\title{
Characterising the secondary maximum in the r-band for Type Ia Supernovae: Diagnostic for the ejecta mass
}

\author{
Seméli Papadogiannakis, ${ }^{1 \star}$ Suhail Dhawan, ${ }^{1}$ Roberta Morosin ${ }^{2}$ and Ariel Goobar ${ }^{1}$ \\ ${ }^{1}$ Department of Physics, The Oskar Klein Centre, Stockholm University, Alba Nova University Centre, SE-106 91 Stockholm, Sweden \\ ${ }^{2}$ Department of Astronomy, Institute for Solar Physics, Stockholm University, Alba Nova University Centre, SE-106 91 Stockholm, Sweden
}

Accepted XXX. Received YYY; in original form ZZZ

\begin{abstract}
An increase in the number of studied Type Ia supernovae (SNe Ia) has demonstrated that this class of explosions has a greater diversity in its observables than was previously assumed. The reasons (e.g. the explosion mechanism, progenitor system) for such a diversity remain unknown. Here, we analyse a sample of $r$-band light curves of SNe Ia, focusing on their behaviour $\sim 2-4$ weeks after maximum light, i.e. the second maximum. We characterise the second maximum by its timing $\left(t_{r_{2}}\right)$ and the integrated flux $\left(\overline{\mathcal{F}}_{r_{2}}\right)$. We find that $t_{r_{2}}$ correlates with the "colour-stretch" parameter $\mathrm{s}_{B V}$, which can be used as a proxy for ${ }^{56} \mathrm{Ni}$ mass, and $\overline{\mathcal{F}}_{r_{2}}$ correlates with the transparency timescale, $\mathrm{t}_{0}$. Using $\overline{\mathcal{F}}_{r_{2}}$ for a sample of 199 SNe from the Palomar Transient Factory and intermediate Palomar Transient Factory, we evaluate a distribution on $t_{0}$ for a sample of SNe Ia found in an "untargeted" survey. Comparing this distribution to the predictions of $t_{0}$ ranges from models we find that the largest overlap in $t_{0}$ values between models and observations is for the sub-Chandrasekhar double detonation models. We also compare our relations between $t_{0}$ and $\overline{\mathcal{F}}_{r_{2}}$ with that from 1-D explosion models of Goldstein \& Kasen (2018) and confirm that $\overline{\mathcal{F}}_{r_{2}}$ can be used as a diagnostic of the total ejecta mass.
\end{abstract}

Key words: supernovae: general

\section{INTRODUCTION}

Type Ia supernovae (SNe Ia) exhibit diverse observable properties (see Hillebrandt, Kromer, Röpke \& Ruiter 2013; Maguire 2017, for a review). SNe Ia show a great diversity in their spectroscopic display (e.g. Blondin, et al. 2012; Folatelli, et al. 2013) and their peak luminosities differ by a factor of 10 (e.g. Suntzeff 1996, 2003; Stritzinger et al. 2006; Li et al. 2011; Taubenberger 2017). The amount of ${ }^{56} \mathrm{Ni}$ (Contardo, Leibundgut \& Vacca 2000) and the total ejected mass (Scalzo et al. 2014) also show a wide dispersion. The dispersion in the ${ }^{56} \mathrm{Ni}$ masses can explain the widthluminosity relation (WLR; Phillips 1993) which is used to correct the SN Ia peak luminosity and use them as distance indicators in cosmology (see Goobar \& Leibundgut 2011; Leibundgut \& Sullivan 2018, for a review).

Spectroscopically normal SNe Ia have optical ( $B$-band) light curves showing a rise to maximum of $\sim 18$ days and a post-maximum decline to an exponentially declining tail. However, at redder wavelengths $(i z Y J H K)$ filters the light

^ E-mail: semeli@fysik.su.se curve morphology is markedly different, showing two maxima (Elias et al. 1981; Hamuy et al. 1996; Meikle 2000). Kasen (2006) explains the emergence of the second maximum as a result of the ionisation transition in the irongroup elements (IGEs) in the ejecta from doubly to singly ionised, leading to a weakening of Fe III and Co III lines and a strengthening of Fe II and Co II lines (see also Blondin, Dessart, \& Hillier 2015). The timing of the second maximum $\left(t_{2}\right)$ is a function of the optical decline rate (Hamuy et al. 1996; Dhawan et al. 2015) indicating that objects with more synthesized ${ }^{56} \mathrm{Ni}$ have a later second maximum. Hence, $t_{2}$ has been used to estimate the ${ }^{56} \mathrm{Ni}$ mass (Dhawan et al. 2016) and to standardize the $\mathrm{SNe}$ for distance measurements in cosmology (Shariff et al. 2016).

In $r$-band, SNe show a weaker second maximum compared the redder filters, akin to a plateau starting at $\sim 15$ days after maximum. The aim of this study is to characterise the features of the $r$-band light curve and to quantitatively search for relations with global properties of the SN explosion, e.g. the total radioactive ${ }^{56} \mathrm{Ni}$ mass and total ejecta mass. This is important also to understand the cause of the $r$-band plateau feature, which has not been studied before. 
With this paper, we aim to fill the gap in light-curve studies with observations in $r$-band.

The onset of large programs to observe samples of SNe Ia at low-redshift $(z<0.1)$ has a led to a large library of $r$-band light curves. Ongoing and recently concluded campaigns, e.g. the Carnegie Supernova Project (CSP-I) (Contreras et al. 2010; Stritzinger et al. 2011), CfA supernova program (Hicken et al. 2009), Palomar Transient Factory (Rau, et al. 2009, PTF;), Carnegie Supernova Project-II (Hsiao et al. 2018; Phillips et al. 2018), Zwicky Transient Facility (ZTF; Graham et al. in preparation), Foundation Supernova Survey (Foley, et al. 2018), have provided and will provide multi-band light curves of SNe Ia. Hence, this investigation is very timely to understand the characteristics of $r$-band light curve features and how they connect to physical properties of SN explosions. Moreover, studies with theoretical light curves, cross-matched with spectra, have postulated a causal mechanism for the $r$-band plateau. Therefore, a study of the observational properties of SNe Ia will be highly complementary to the existing theoretical work.

The structure of this paper is as follows: in Section 2 we describe the datasets used in this study and in Section 3 we detail the methodology for fitting the $r$-band light curves. We present our results in Section 4 and finally discuss them and conclude in Section 5 and Section 6 .

\section{DATA}

In this investigation, we analyse correlations between wellunderstood decline parameters, bolometric light curve properties and $r$-band properties at late times (note that in this paper late times refers to epochs later than 10 days after maximum light). We use data from the Carnegie Supernova Project (CSP-I) (Contreras et al. 2010; Stritzinger et al. 2011) and CfA supernova program (Hicken et al. 2009) for analysing the correlations between the different light curve properties.

The Palomar Transient factory (PTF) and its successor the intermediate transient factory (iPTF) were large fieldof-view transient surveys that discovered and obtained light curves for hundreds of SNe Ia (see Papadogiannakis et al. 2019, for details). Unlike the CSP and CfA, which were exclusively follow-up campaigns, PTF/iPTF also discovered the SNe, giving a better control on the selection effects in finding them, important for characterising properties for a population of SNe Ia (Papadogiannakis et al. 2019). The drawback of this dataset is that it mostly has photometry in one filter, which is the $R$-band for a large fraction of the $\mathrm{SNe}$. Since we want to characterise the $r$-band second maximum, we only use data for $\mathrm{SNe}$ in the phase range +10 to +40 days (an example is shown in Figure 1).

A summary of the SNe used in this study along with their derived properties of the secondary maximum is presented in the Appendix A.

\section{ANALYSIS}

In this section, we describe the analysis method for evaluating the parameters in the study. We use two different methods to probe the plateau or secondary maximum in the $r$-band where we determine the time of the plateau $\left(t_{r_{2}}\right)$ and calculate the integrated normalised flux around the dates of the plateau $\left(\overline{\mathcal{F}}_{r_{2}}\right)$. We use the same symbols for both the $r$-band and $R$-band used with the CSP-I, CfA and PTF and iPTF survey data respectively. In this analysis, we explore whether these properties of the $r$-band plateau correlate with the global properties of $\mathrm{SNe} \mathrm{Ia}$, e.g. total radioactive nickel mass, total ejecta mass. In previous studies it has been shown that the ordering parameter $\mathrm{s}_{B V}$ (Burns et al. 2014) correlates with the peak bolometric luminosity (Hoeflich, et al. 2017). Arnett's rule states that the instantaneous rate of energy deposition from radioactive decay equals the output flux at maximum (Arnett 1982). Hence, we use the $\mathrm{s}_{B V}$ parameter as a proxy for the ${ }^{56} \mathrm{Ni}$ mass in our analyses. In previous studies, it has been shown that the transparency timescale $\left(t_{0}\right)$, i.e. the epoch at which the ejecta optical depth is unity, is directly related to the total ejecta mass (Jeffery 1999; Stritzinger et al. 2006).

Hence, we test for correlations between the properties of the plateau with the ordering parameter $\mathrm{s}_{B V}$ and the transparency timescale. Below, we describe the method for evaluating the properties of the plateau, $t_{r_{2}}$ and $\overline{\mathcal{F}}_{r_{2}}$, as well as the transparency timescale. The ordering parameter $\mathrm{s}_{B V}$ is calculated using the "colour model" in the SNooPy light curve fitting software (Burns et al. 2014).

\subsection{Time of secondary maximum}

To estimate the time of the plateau or secondary bump in $r$-band, $t_{r_{2}}$, we first run Gaussian processes (GP) with a Matérn $\frac{2}{3}$ kernel to get the most likely function, the latent function $\ell$, that describes the data and its uncertainty.

Gaussian processes is a non-parametric way of predicting the underlying function behind data and works well with unevenly spaced data, such as the one we have. It is also able to predict an error for each given part of the function which sets it apart from other techniques.

To accommodate the GP priors we normalise the fluxes so that the mean error is 1 and compute $t_{r_{2}}$ in flux space. To get a better understanding of the error of the $t_{r_{2}}$ estimate we then perturb the data points within their errors 100 times using Monte Carlo simulations and get 100 latent functions. We then compute the derivative of the sampled functions with respect to time and choose the $t_{r_{2}}$ as a point with zero derivative and negative second derivative that lies between day +13 and +40 with respect to maximum light. Using the results of this we can then determine the probability that we have encountered a maximum, a shoulder or neither for each SN. If there is an inflection point rather than a bump we calculate the point of inflection and call it a shoulder.

In Figure 1 an example fit is shown. We used the same Monte Carlo simulations to estimate the error of $t_{r_{2}}$. In our final sample we require the $\mathrm{SNe}$ to have at least 4 data points within the times +10 and +40 days with respect to maximum light and a standard deviation of the $t_{\max }$ of less than 1.6 days. All SNe rejected by these criteria were visually inspected to make sure no good fit was rejected. This leaves $112 \mathrm{SNe}$ from CfA, $70 \mathrm{SNe}$ from CSP and $240 \mathrm{SNe}$ from PTF and iPTF for which a $t_{r_{2}}$ measurement could be obtained. 


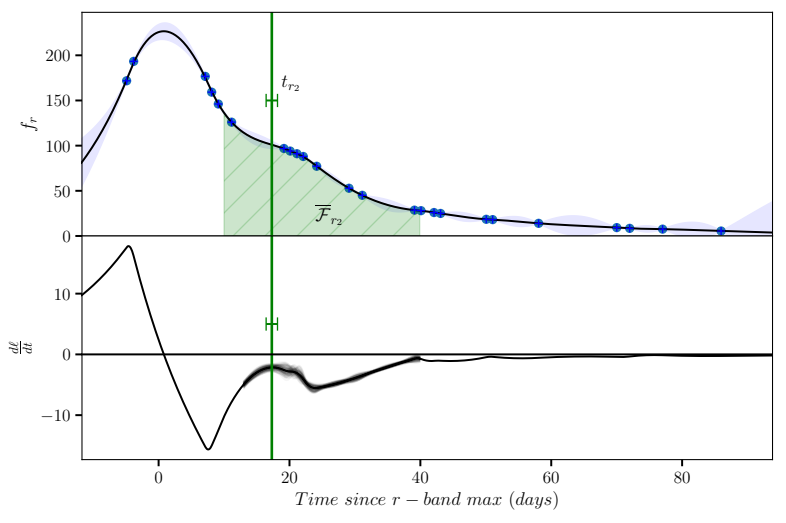

Figure 1. In the upper panel we show an example of the Gaussian processes (GP) fit and estimation of the bump time, $t_{r_{2}}$ with the error estimate coming from Monte Carlo (MC) simulations. The green shaded green area shows the estimate of the $\overline{\mathcal{F}}_{r_{2}}$. In the lower panel the curve shows the derivative of the most likely function given by GP and the dispersion of the derivative in grey lines. The green solid line shows the estimation of $t_{r_{2}}$ averaged over all MC runs.

\subsection{Integrated flux under the secondary maximum}

Another way that we quantify the r-band secondary maximum is using the mean normalised flux integrated in the interval +15 and +40 days with respect to maximum light $\left(\overline{\mathcal{F}}_{r_{2}}\right)$. This metric to quantify the second maximum was proposed by Krisciunas, et al. (2001) for the $i$-band, and here, we adapt it for the $r$-band. We calculate $\overline{\mathcal{F}}_{r_{2}}$ by integrating the latent function from heteroscedastic GP of the rest-frame light-curve normalised to the peak flux and divided by the number of days of the interval. By using GP we get a data driven estimate of the error that allows a robust measurement even when there are gaps in the data. We require at least 4 data points within the integration interval. We get measurements of $\overline{\mathcal{F}}_{r_{2}}$ of $61 \mathrm{SNe}$ from CfA, 53 SNe from CSP and $199 \mathrm{SNe}$ from PTF and iPTF. The two parameters, $\overline{\mathcal{F}}_{r_{2}}$ and $t_{r_{2}}$ are related as seen in figure 2 .

\subsection{Transparency timescale}

In previous studies (Jeffery 1999; Stritzinger et al. 2006), it has been shown that the transparency timescale, i.e. the epoch at which the ejecta have optical depth of unity, is a proxy for $\mathrm{M}_{\mathrm{ej}}$. The transparency timescale is evaluated from the bolometric light curve by fitting a radioactive decay energy (RDE) deposition curve to the tail of the observations. We use a standard least squares fitting methodology in this analysis. We add an error of 2 days to the error from the fit of $t_{0}$, corresponding to the average error in inferring the rise time of an SN Ia (see Scalzo et al. 2014, for details). The bolometric light curve is created from the multi-band photometry using the method described in Dhawan, et al. (2018). We convert the observed magnitudes to de-reddened fluxes and interpolate the filters onto the same time step. The fluxes are integrated using the trapezoidal rule and then converted to absolute luminosities using the observed dis-

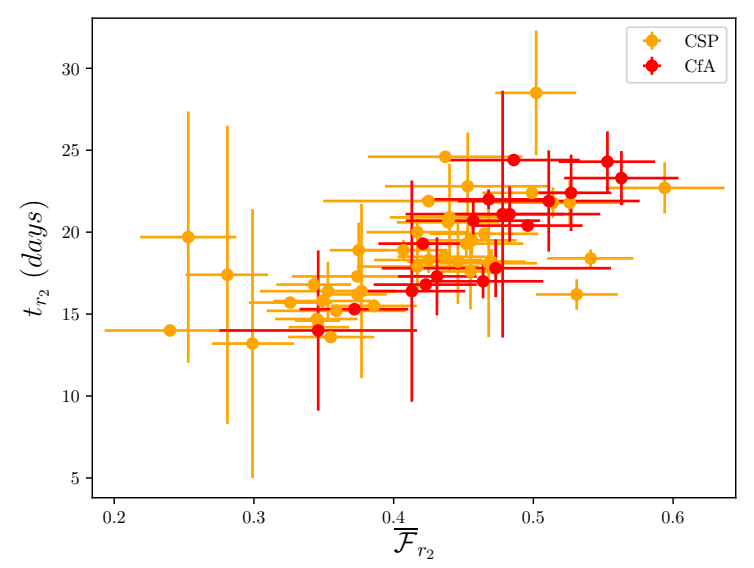

Figure 2. Time of the bump, $t_{r_{2}}$ versus $\overline{\mathcal{F}}_{r_{2}}$ the two parameters measured at the secondary maximum of the r-band light-curve.

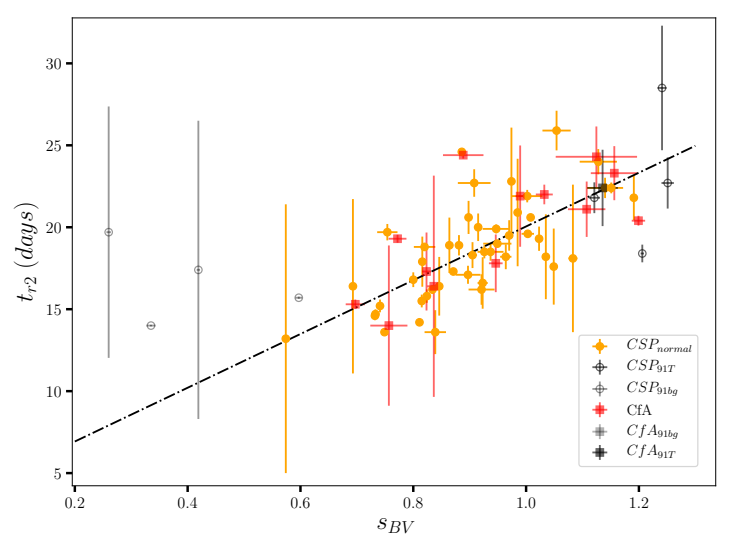

Figure 3. Time of the bump, $t_{r_{2}}$ versus $\mathrm{s}_{\boldsymbol{B} V}$ for the $\mathrm{SNe}$ from CSP and CfA. The dashed line shows the best fit straight line with a Spearman $r=0.7$ and a $p-v a l u e<10^{-11}$. The gray data points show the spectroscopic outliers such as $91 \mathrm{bg}$ and $91 \mathrm{~T}-\mathrm{like}$ SNe from both surveys.

tances (e.g., see Contardo, Leibundgut \& Vacca 2000). The expression for the RDE deposition curve is given as follows:

$$
\begin{aligned}
E_{\mathrm{dep}}=E_{\mathrm{Ni}}+E_{\mathrm{Co} \mathrm{e}^{+}}+\left[1-\exp \left(-\tau_{\gamma}\right)\right] E_{\mathrm{Co} \gamma} \\
=\lambda_{\mathrm{Ni}} \mathrm{N}_{\mathrm{Ni} 0} \exp \left(-\lambda_{\mathrm{Ni}} \mathrm{t}\right) \mathrm{Q}_{\mathrm{Ni} \gamma} \\
+\lambda_{\mathrm{Co}} \mathrm{N}_{\mathrm{Ni} 0} \frac{\lambda_{\mathrm{Ni}}}{\lambda_{\mathrm{Ni}}-\lambda_{\mathrm{Co}}}\left[\left[\exp \left(-\lambda_{\mathrm{Co}} \mathrm{t}\right)-\exp \left(-\lambda_{\mathrm{Ni}} \mathrm{t}\right)\right]\right. \\
\left.\times\left\{Q_{\mathrm{Co}^{+}}+Q_{\mathrm{Co} \gamma}\left[1-\exp \left(-\tau_{\gamma}\right)\right]\right\}\right],
\end{aligned}
$$

where the factor $\left(1-\exp \left(-\tau_{\gamma}\right)\right)$ is replaced by 1 for ${ }^{56} \mathrm{Ni}$ since complete trapping of $\gamma$-rays occurs at early times, when most of the light curve is powered by ${ }^{56} \mathrm{Ni}$. $\lambda_{\mathrm{Ni}}$ and $\lambda_{\mathrm{Co}}$ are the efolding decay times of 8.8 days and 111.3 days for ${ }^{56} \mathrm{Ni}$ and ${ }^{56} \mathrm{Co}$ respectively. $Q_{\mathrm{Ni} \gamma}(1.75 \mathrm{MeV})$ is the energy release 

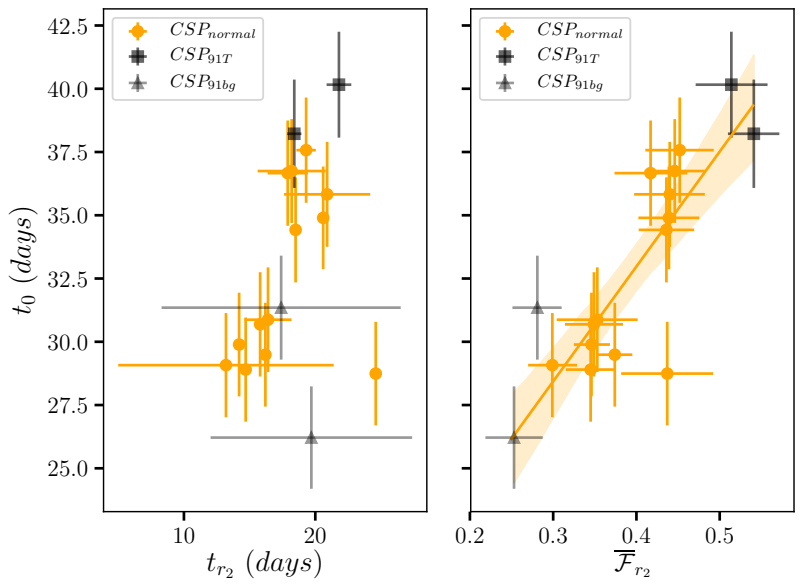

Figure 4. Left: Transparency timescale $t_{0}$ vs. bump time, $t_{r_{2}}$ both in units of days. Right: Integrated flux, $\overline{\mathcal{F}}_{r_{2}}$, versus $t_{0}$ for the CSP sample, the solid line shows the best fit line and the shaded region the error of the slope. Note that the errorbars in $t_{0}$ are a systematic error of 2 days from the uncertainty of the rise-time of Type Ia SNe.

per ${ }^{56} \mathrm{Ni} \rightarrow{ }^{56} \mathrm{Co}$ decay. $Q_{\mathrm{Co} \gamma}(3.61 \mathrm{MeV})$ and $Q_{\mathrm{Co} e^{+}}(0.12$ $\mathrm{MeV})$ are the $\gamma$-ray and positron energies, respectively, released per ${ }^{56} \mathrm{Co} \rightarrow{ }^{56} \mathrm{Fe}$ decay (see Stritzinger et al. 2006). Equation 1 is only applicable in the optically thin limit, when the thermalized photons can freely escape.

$\tau_{\gamma}$ is the mean optical depth, calculated by integrating from the point of emission to the surface of the ejecta (see Jeffery 1999, for a derivation of the expression). It has a simple $\mathrm{t}^{-2}$ dependence, given as,

$\tau_{\gamma}=\frac{t_{0}^{2}}{t^{2}}$

where $t_{0}$ is the transparency timescale, which by construction in Jeffery (1999) is the epoch at which the optical depth is unity.

\section{RESULTS}

The timing of the NIR second peak has been shown to correlate with light curve properties relating to the peak absolute brightness (e.g. decline rate, $\Delta m_{15}$ ) (Hamuy et al. 1996; Folatelli, et al. 2010; Biscardi, et al. 2012; Dhawan et al. 2015). Here, we investigate whether the $r$-band bump shows any significant trends with similar light curve parameters. Burns et al. (2014) demonstrated that the $\Delta m_{15}$ light curve shape parameter does not adequately capture the diversity of SNe Ia, especially at the faint end, where the SNe transition to the exponential decline at $<15$ days from maximum light. As an alternative, they propose a "colour-stretch" parameter $\mathrm{s}_{B V}$, which is more accurate at ordering even the faint end of the observed distribution of SNe Ia properties. Previous studies have shown that $\mathrm{s}_{B V}$ is correlated strongly with bolometric properties e.g. $\mathrm{L}_{\max }$ (Dhawan, Leibundgut, Spyromilio \& Blondin 2017; Hoeflich, et al. 2017) and hence, a strong indicator of global properties like ${ }^{56} \mathrm{Ni}$
(Arnett 1982). Thus, we use $\mathrm{s}_{B V}$ as a proxy for the intrinsic luminosity of the SN.

In Figure 3, we plot the resulting correlation between $t_{r_{2}}$ and $\mathrm{s}_{B V}$ and find a strong correlation between the two quantities (Spearman $r=0.7$ and a $p$-value $<10^{-11}$ ). This indicates that $\mathrm{SNe}$ with a later $t_{r_{2}}$ are intrinsically brighter, similar to the behaviour for the equivalent feature at redder wavelengths (Hamuy et al. 1996; Folatelli, et al. 2010; Dhawan et al. 2015). Hence, $t_{r_{2}}$ can be used as a possible luminosity indicator. However when we look at how $t_{r_{2}}$ correlates with the Hubble residuals in the Mould $R$-band from the PTF and iPTF sample we find no correlation suggesting that at least in the $R$-band, $t_{r_{2}}$ is not a good predictor for luminosity.

The above correlation relates $t_{r_{2}}$ features to the intrinsic luminosity in the B-band. We investigate whether $t_{r_{2}}$ also correlates with observables relating to progenitor properties, e.g. the total ejected mass. Previous studies have noted that the transparency time-scale $\left(t_{0}\right)$ of the bolometric light curve can be an indicator for the ejecta mass (Jeffery 1999; Stritzinger et al. 2006; Scalzo et al. 2014; Dhawan, Leibundgut, Spyromilio \& Blondin 2017) with longer timescales corresponding to higher masses. The transparency time-scale is the epoch when the optical depth of the SN ejecta is unity. We derive it from the bolometric light curve by fitting a radioactive decay energy (RDE) deposition curve to the tail ( +40 to +90 days) of the light curve (see Jeffery 1999; Stritzinger et al. 2006; Scalzo et al. 2014, for details).

Due to the stringent cuts on the data to sample the peak in $u \rightarrow H$ filters, the final sample of SNe with $\overline{\mathcal{F}}_{r_{2}}$ and $t_{0}$ measurements is comparatively smaller and coming from the CSP sample only. We note that the small size of the dataset shows the importance of having a parameter, derived only from a single filter, that correlates with $t_{0}$ to derive $t_{0}$ values for a large sample of SNe. We find a significant correlation between the transparency time-scale, $t_{0}$ and the integrated flux as indicated in Figure 4 with a Spearman $R=0.8$ and a p-value of $<10^{-5}$. The best fit parameters are:

$t_{0}=44.92( \pm 5.86) \times \overline{\mathcal{F}}_{r_{2}}+15.00( \pm 2.32)$,

Since the PTF and iPTF data have a series of well sampled $R$-band light curves (Papadogiannakis et al. (2019)) from an untargeted survey, we can apply Equation 3 to a large dataset where the lack of multi-band data would have otherwise prevented us from deriving $t_{0}$ in the absence of the above relation. We get an extrapolated distribution of $t_{0}$ shown in Figure 5 . The uncertainty on the derived $t_{0}$ for each object is $\sim 3$ days, we expect that a larger sample of $\mathrm{SNe}$ for deriving the best fit relation will decrease this error.

In previous works a direct comparison of the model predictions for $t_{0}$ versus other global properties (e.g. ${ }^{56} \mathrm{Ni}$ mass) has been important to suggest that multiple progenitor channels could be contributing to the observed diversity of SNe Ia (Scalzo et al. 2014; Childress, et al. 2015; Wygoda, Elbaz \& Katz 2017; Dhawan, et al. 2018). Additionally, studies have compared different properties of SNe Ia (e.g. brightness, ${ }^{56} \mathrm{Ni}$ mass) with the predictions from models (Ruiter, et al. 2013; Piro, Thompson \& Kochanek 2014). We present the distribution of $t_{0}$, such that it can be compared with theoretical predictions for specific model scenarios to distinguish between the different possibilities for 


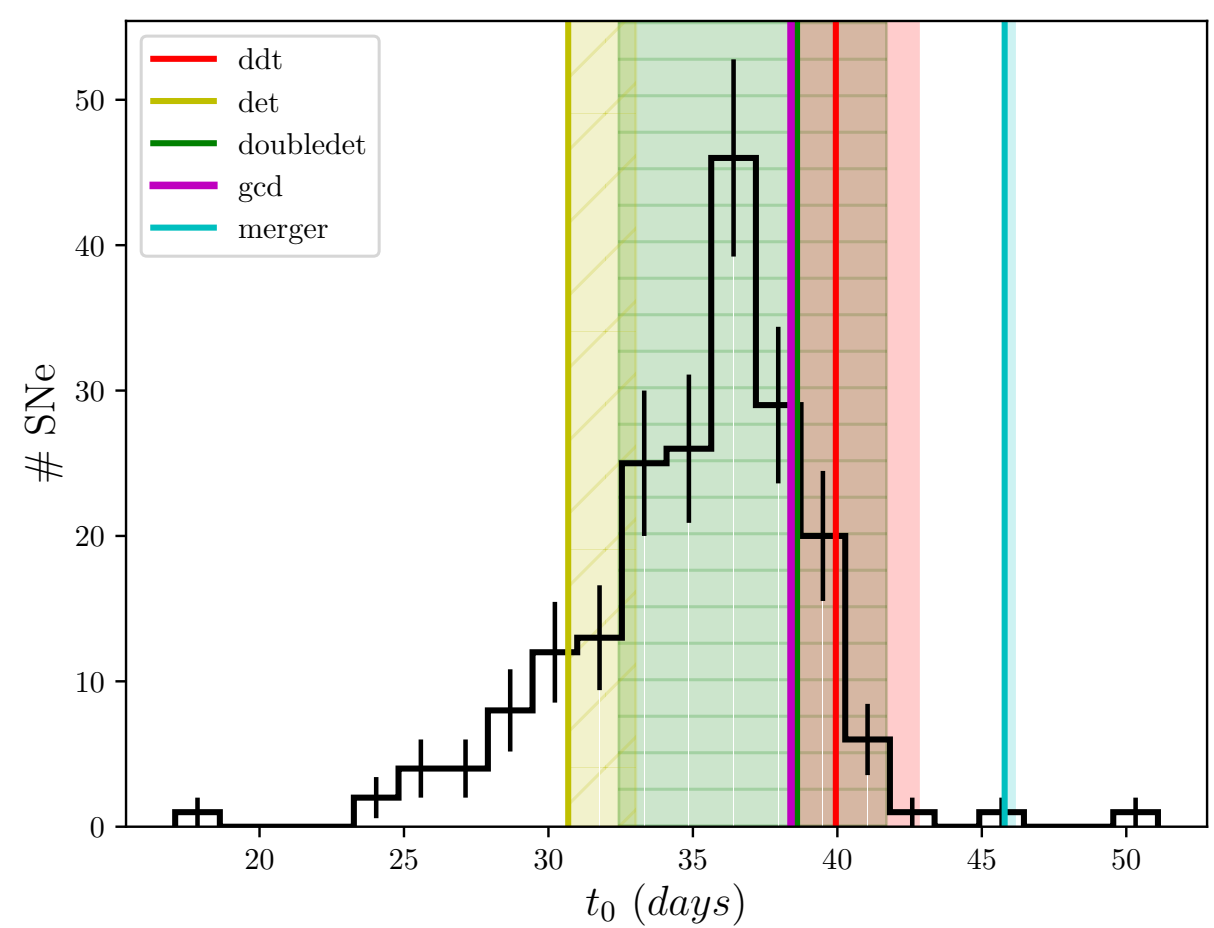

Figure 5. This is the extrapolated transparency time-scale $t_{0}$ distribution of the $199 \mathrm{PTF}$ and iPTF SNe based on the correlation derived from the CSP sample, the errorbars show the Poisson error in each bin. The different shaded regions represent different model prediction of $t_{0}$.

the origin of SNe Ia. In Figure 5 we overplot the ranges of $\mathrm{t}_{0}$ from a range of different models taken from the Heidelberg Supernova Model Archive Kromer, et al. (2017). The models represented are the pure detonations (det) from Sim, et al. (2010), "double detonation" (doubledet) from Fink et al. (2010), gravitationally confined detonation (gcd) from Seitenzahl et al. (2016) and violent merger models (merger) from Pakmor et al. (2010) and Pakmor et al. (2012). Since the uncertainty on the inferred $t_{0}$ for the SNe from PTF and iPTF is of order a few days, we do not compare individual SNe to model predictions, but rather compare the range of $t_{0}$ values observed to the predicted ranges for the different model grids. We note that no model covers the entire distribution but the "double detonation" (doubledet) model is that that covers the largest part of the distribution and the violent merger models cover the least. In Table 1 we show which percentage of the distribution is covered by each of the tested models.

While our focus here is on the $r$-band, the method can be applied to other filters as well. We apply the same analysis for the $i$-band data of CSP and CfA and find similar correlations as seen in figure 6 and 7 .

We note that the dispersion in the $t_{i_{2}}$ vs. $\mathrm{s}_{B V}$ relation is tighter in the $i$-band than in the $r$-band whereas the relation between $t_{0}$ and $\overline{\mathcal{F}}_{i_{2}}$ has similar scatter. This reproduction of correlations seems to indicate a common origin of the $r$ - and $i$-band secondary maximum as discussed in Kasen (2006).

In Figure 8 we show the correlation between the $i$-band and the $r$-band integrated flux, $\overline{\mathcal{F}}_{i_{2}}$ and $\overline{\mathcal{F}}_{r_{2}}$.

\begin{tabular}{lc}
\hline Model & $\begin{array}{c}\text { Fraction } \\
(\%)\end{array}$ \\
\hline Sub-Chandra Double Detonaton (Doubledet) & 77.4 \\
Chandrasekhar mass Delayed Detonation (ddt) & 17.6 \\
Sub-Chandra Detonation (Det) & 11.6 \\
Violent Merger (Merger) & 0.5 \\
\hline
\end{tabular}

Table 1. The percentage of $\mathrm{SNe}$ in the distribution from 5 for the different models tested coming from the Heidelberg Supernova Model Archive Kromer, et al. (2017). The gravitationally confined detonations (gcd) scenario is not listed above since it only has one model and hence, we cannot calculate a range of overlap.

\section{DISCUSSION}

We find that $t_{r_{2}}$ are correlated with $\mathrm{s}_{B V}$ as well as the transparency timescale, indicating its link to fundamental properties of the $\mathrm{SNe}$, e.g. luminosity, ejecta mass. We derive equivalent relations for the models and compare them to the observations.

From the transparency timescale, $t_{0}$, that we derived earlier, we can get the total ejecta mass $\left(\mathrm{M}_{\mathrm{ej}}\right)$ using the equation (Jeffery 1999; Stritzinger et al. 2006; Dhawan, Leibundgut, Spyromilio \& Blondin 2017; Dhawan, et al. 2018); 

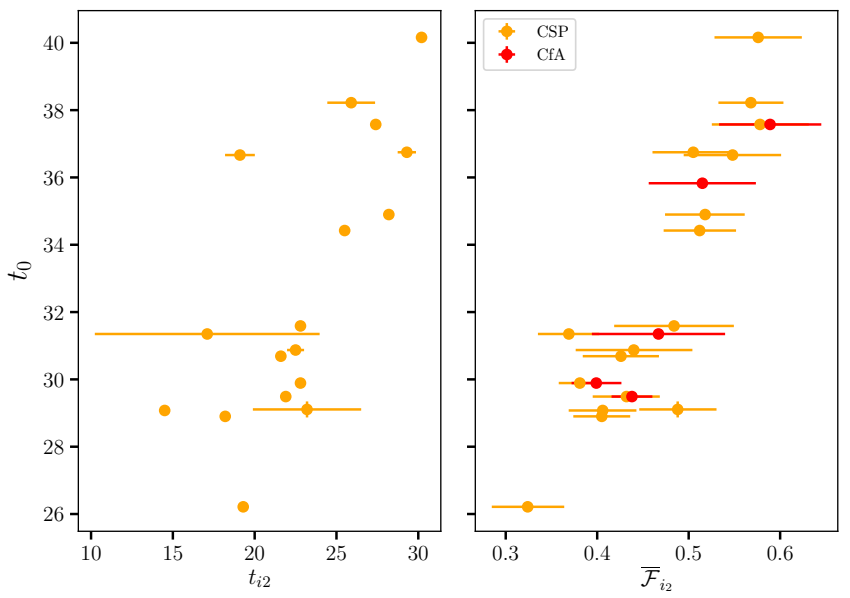

Figure 6. I the left panel we show $t_{0}$ vs. $t_{i_{2}}$ and in the right panel $t_{0}$ vs. $\overline{\mathcal{F}}_{i_{2}}$. We note similar correlations as seen for r-band.

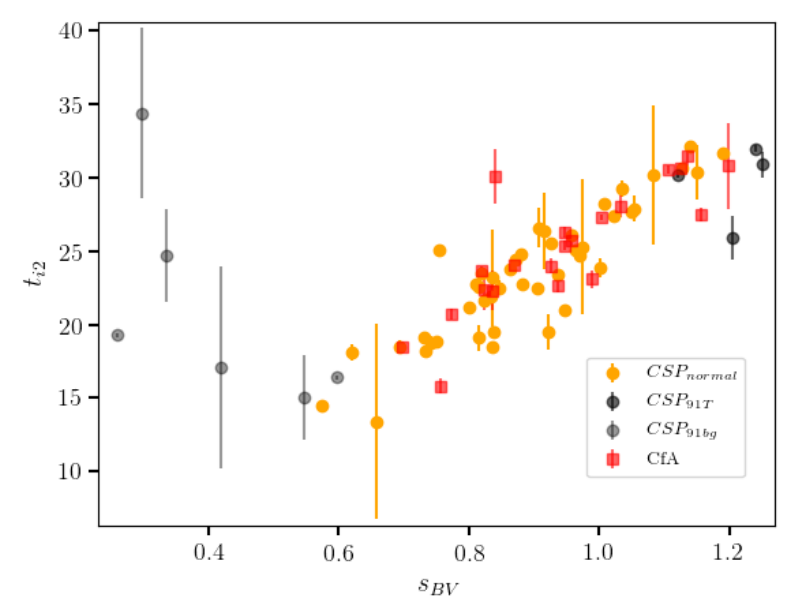

Figure 7. Here we show the relation between $\mathrm{s}_{\boldsymbol{B} V}$ and $t_{\boldsymbol{i}_{2}}$ that is tighter than that in the r-band.

$M_{\mathrm{ej}}=1.38 \cdot\left(\frac{1 / 3}{q}\right) \cdot\left(\frac{v_{e}}{3000 \mathrm{kms}^{-1}}\right)^{2} \cdot\left(\frac{t_{0}}{36.80 \text { days }}\right)^{2} \mathrm{M}_{\odot}$.

for the PTF and iPTF sample. This equation describes the capture rate of $\gamma$ rays in an expanding spherical volume for a given distribution of the radioactive material. Here, $v_{e}$ is the e-folding velocity, which provides the scaling length for the expansion and $q$ represents the distribution of the ${ }^{56} \mathrm{Ni}$ in the ejecta. The range of expected $\mathrm{v}_{\boldsymbol{e}}$ values is between $\sim 2600$ and $3200 \mathrm{~km} \mathrm{~s}^{-1}$ with a typical value for a normal $\mathrm{SN}$ Ia being $\sim 3000 \mathrm{~km} \mathrm{~s}^{-1}$. For the value of $q, 1 / 3$ implies uniformly distributed ejecta with higher values, till a maximum of unity, implying progressively more centrally concentrated ${ }^{56} \mathrm{Ni}$. For the comparison with the relations from the model described below we use $v_{e}=3000 \mathrm{~km} / \mathrm{s}$ and $q=1 / 3$, typical values for a normal SN Ia (see Figure 9 . As in pre-

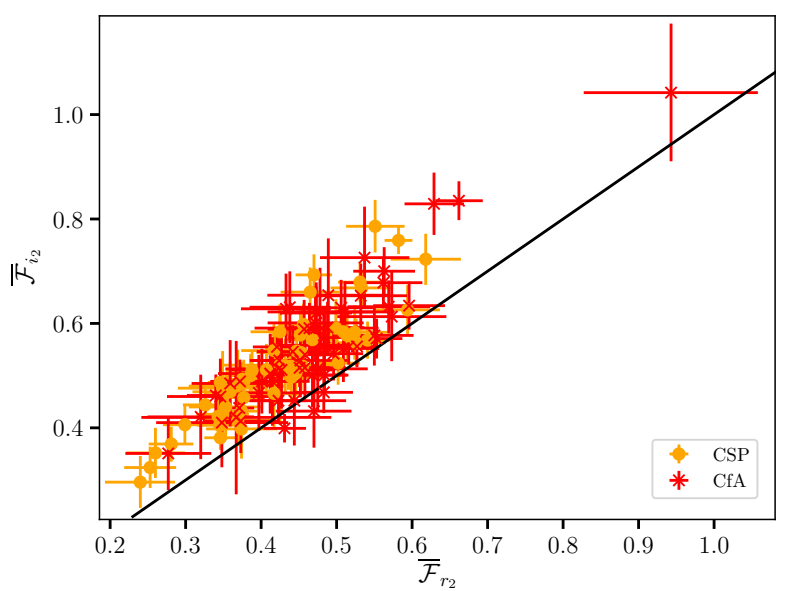

Figure 8. $t_{r_{2}}$ in days against $\overline{\mathcal{F}}_{r_{2}}$ with the sub-classified SNe in i-band of each survey with different symbols and colours as indicated. The solid black line shows the directly proportional equation between the two parameters.

vious studies, we assume a constant $\gamma$-ray opacity of 0.025 $\mathrm{cm}^{2} \mathrm{~g}^{-1}$ (Swartz, Sutherland \& Harkness 1995).

When comparing with radiative transfer model from Goldstein \& Kasen (2018) we find a strong correlation between $t_{0}$ and $\overline{\mathcal{F}}_{r_{2}}$ suggesting that bump features in the $r$-band can be directly connected to the ejecta mass $\mathrm{M}_{\mathrm{ej}}$. We analyse a grid of 4500 state-of-the-art radiative transfer models from Goldstein \& Kasen (2018) which span a large range of the physical parameters of SNe Ia, e.g. kinetic energy, total mass, radioactive nickel mass. We compute synthetic photometry in the SDSS- $r$ band and evaluate $t_{r_{2}}$ and $\overline{\mathcal{F}}_{r_{2}}$ values for the models. The aim is to note whether the models directly show a correlation between $\mathrm{M}_{\mathrm{ej}}$ (which is $\mathrm{M}_{W D}$ for SNe Ia since the complete white dwarf (WD) is unbound in the explosion) and $t_{r_{2}}$ or $\overline{\mathcal{F}}_{r_{2}}$, and hence, confirm or refute using theoretical predictions whether the $r$-band bump can be used to derive physical properties for large samples of SNe Ia. We note that the models of Goldstein \& Kasen (2018) are computed under the assumption of local thermodynamic equilibrium (LTE), which does not hold true for the phase of the light curve probed by the bump (see Goldstein \& Kasen (2018)), however, they can be used to determine relations between the parameters.

While we find that the bump like feature in the model is more pronounced than in the data and hence, cannot be used for robust estimates of the theoretical values of $t_{r_{2}}$ and $\overline{\mathcal{F}}_{r_{2}}$, we can use the inferred values for understanding correlations between the parameters. For models with $\mathrm{M}_{W D}>0.5$ $\mathrm{M}_{\odot}$ there is a strong correlation between $\mathrm{M}_{W D}$ and $t_{r_{2}}$. Explosion modelling has shown that carbon-oxygen WDs of masses $\gtrsim 0.7 \mathrm{M}_{\odot}$ leads to an SN Ia, the low limit on the mass of the exploding WD. Hence, for comparing observations to models, we look at the mass range $\left(\mathrm{M}_{W D}>0.7\right.$ $\left.\mathrm{M}_{\odot}\right)$.

We also find that the $\mathrm{M}_{W D}$ value is correlated with $\overline{\mathcal{F}}_{r_{2}}$ (Figure 9$)$. This provides corroborating evidence $\overline{\mathcal{F}}_{r_{2}}$ values can be used as a diagnostic of the $\mathrm{M}_{\mathrm{ej}}$ for SNe Ia. This 


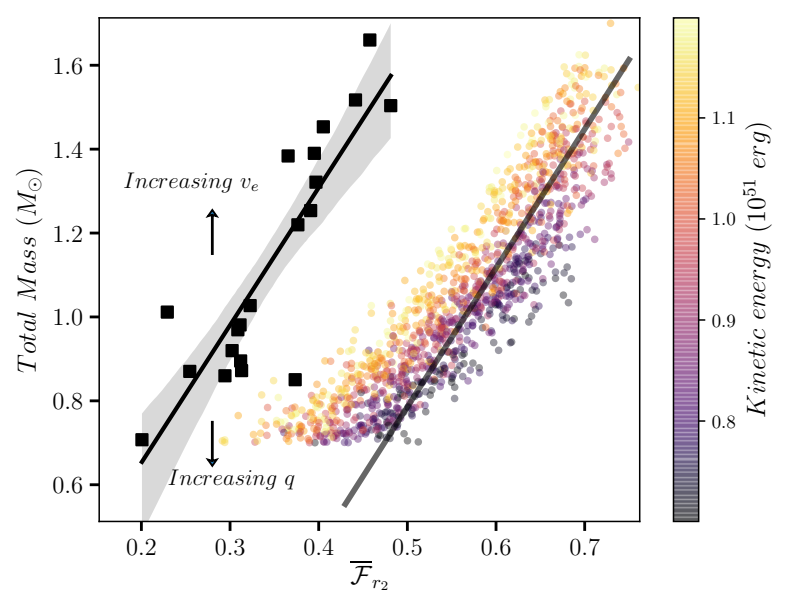

Figure 9. The total mass vs. $\overline{\mathcal{F}}_{r_{2}}$ from the Goldstein \& Kasen (2018) models. We limit the models shown to the ones with kinetic energy between $0.7-1.2 \times 10^{51}$ erg since that is the physically motivated limits (e.g. Blondin et al. 2017). For comparison we have plotted the total mass vs. $\overline{\mathcal{F}}_{r_{2}}$ for the data (black squares). We derived the total mass from the $t_{0}$ using typical values of $v_{e}$ and $q$ from explosion models (see text for more details). The best fit line from the data is shifted using the intercept from the models but keeping the same slope (black line). This shows that the model and the data find similar trends between the total mass and $\overline{\mathcal{F}}_{r_{2}}$

in turn means that we can probe the $\mathrm{M}_{\mathrm{ej}}$ for higher redshifts compared to a similar analysis in the $i$-band in surveys such as LSST to get an estimate of the $\mathrm{M}_{\mathrm{ej}}$ for higher redshifts provided a minimal cadence of 5 restframe days in order to get a $t_{r_{2}}$ and $\overline{\mathcal{F}}_{r_{2}}$ measurement. However, it is important to note that the range of the two free parameters in equation 4 is large and when converting to $\mathrm{M}_{\mathrm{ej}}$ this gives rise to such large uncertainties that it is not possible to accurately quantify the best fit values. We do see a qualitative trend for both the data and the models from Goldstein \& Kasen (2018) in the same direction but not with the same values.

\section{CONCLUSIONS}

In this paper we present two methods of measuring the secondary maximum of light-curves in the $r$-band that also work for redder bands, e.g. $i$-band. We then used the Carnegie Supernova Project (CSP-I) (Contreras et al. 2010; Stritzinger et al. 2011) and CfA supernova program (Hicken et al. 2009) to derive correlations that we applied to the light-curve sample of PTF and IPTF (Papadogiannakis et al. 2019) to show the distribution of the transparency timescale which is a proxy for total mass. Since the distribution comes from an untargeted survey it represents more closely the true distribution of SNe Ia since the bias of finding SNe in larger galaxies is limited.

In summary our main conclusions are:

- We find a significant correlation between $t_{r_{2}}$ and $\mathrm{s}_{B V}$ which suggest brighter SN have a later bump in the $r$-band, since $\mathrm{s}_{B V}$ correlates with the peak brightness of the $\mathrm{SN}$ and ${ }^{56} \mathrm{Ni}$ mass.

- There is a significant correlation between $\overline{\mathcal{F}}_{r_{2}}$ and the transparency timescale, $t_{0}$ which is a measure of when the SN ejecta becomes optically thin and is a probe for the total mass of the SN Ia explosion.

- These correlations suggest that SN physics can be extracted from the secondary maximum in r-band just as the secondary maximum in the NIR and IR, but with more accessible resources and less observing time.

- We also find a correlation between $\overline{\mathcal{F}}_{r_{2}}$ and total mass when examining the light-curves in the r-band from the Goldstein \& Kasen (2018) models for masses larger than $0.3 M_{\text {solar }}$. We note that the shape of the r-band lightcurves in the models do not agree with our observations and that the LTE approximations and thus it is only reasonable to compare the trend which is in agreement with our data. More accurate modelling is necessary to further investigate the correlation between $\overline{\mathcal{F}}_{r_{2}}$ and total mass.

- We see a correlation between the total mass from the modes from Goldstein \& Kasen (2018) and $\overline{\mathcal{F}}_{r_{2}}$ strengthening the suggestion that $\overline{\mathcal{F}}_{r_{2}}$ is a probe of the total mass.

- When doing the same analysis in the $i$-band similar correlations are found.

- We present a distribution of the transparency timescale $\mathrm{t}_{0}$ from PTF and iPTF and compare it to models. We find that no model covers the entire range of values from the observations.

\section{ACKNOWLEDGEMENTS}

We would like to thank D. Goldstein for sending us the model files. We acknowledge support from the Swedish National Science Council as well as the Swedish National Space Agency.

\section{REFERENCES}

Arnett W. D., 1982, ApJ, 253, 785

Biscardi I., et al., 2012, A\&A, 537, A57

Blondin S., et al., 2012, AJ, 143, 126

Blondin S., Dessart L., Hillier D. J., 2015, MNRAS, 448, 2766

Blondin S., Dessart L., Hillier D. J., Khokhlov A. M., 2017, MNRAS, 470, 157

Burns C. R., et al., 2014, ApJ, 789, 32

Childress M. J., et al., 2015, MNRAS, 454, 3816

Contardo G., Leibundgut B., Vacca W. D., 2000, A\&A, 359, 876

Contreras C., et al., 2010, AJ, 139, 519

Dhawan S., Leibundgut B., Spyromilio J., Maguire K., 2015, MNRAS, 448, 1345

Dhawan S., Leibundgut B., Spyromilio J., Blondin S., 2016, A\&A, $588, \mathrm{~A} 84$

Dhawan S., Leibundgut B., Spyromilio J., Blondin S., 2017, A\&A, 602, A118

Dhawan S., et al., 2018, MNRAS, 480, 1445

Elias, J. H., Frogel, J. A., Hackwell, J. A., Persson, E. E., 1981, ApJ, 251, L13

Fink M., Röpke F. K., Hillebrandt W., Seitenzahl I. R., Sim S. A., Kromer M., 2010, A\&A, 514, A53

Folatelli G., et al., 2010, AJ, 139, 120

Folatelli G., et al., 2013, ApJ, 773, 53

Foley R. J., et al., 2018, MNRAS, 475, 193 
Goldstein D. \& Kasen D., 2018, ApJ, 852, L33

Goobar A., Leibundgut B., 2011, ARNPS, 61, 251

Hamuy M., Phillips M. M., Suntzeff N. B., Schommer R. A., Maza J., Smith R. C., Lira P., Aviles R., 1996, AJ, 112, 2438

Hicken M., et al., 2009, ApJ, 700, 331H

Hillebrandt W., Kromer M., Röpke F. K., Ruiter A. J., 2013, FrPhy, 8, 116

Hoeflich P., et al., 2017, ApJ, 846, 58

Hsiao E. Y., et al., 2018, arXiv, arXiv:1810.08213

Jack D., Baron E., Hauschildt P. H., 2015, MNRAS, 449, 3581

Jeffery D. J., 1999, astro, arXiv:astro-ph/9907015

Kasen D., 2006, ApJ, 649, 939

Krisciunas K., et al., 2001, AJ, 122, 1616

Kromer M., et al., 2017, MmSAI, 88, 312K

Leibundgut B., Sullivan M., 2018, SSRv, 214, 57

Li W., et al., 2011, MNRAS, 412, 1441

Livio M., Mazzali P., 2018, PhR, 736, 1

Maguire K., 2017, Handbook of Supernovae, 293-316

Meikle W. P. S., 2000, MNRAS, 314, 782

Pakmor R., Kromer M., Röpke F. K., Sim S. A., Ruiter A. J., Hillebrandt W., 2010, Nature, 463, 61

Pakmor R., Kromer M., Taubenberger S., et al., 2012, ApJ, 747, L10

Papadogiannakis et al., 2019, MNRAS, 483, 5054P

Phillips M. M., et al., 2018, arXiv, arXiv:1810.09252

Phillips M. M., 1993, ApJ, 413, L105

Piro A. L., Thompson T. A., Kochanek C. S., 2014, MNRAS, 438, 3456

Rau A., et al., 2009, PASP, 121, 1334

Ruiter A. J., et al., 2013, MNRAS, 429, 1425

Scalzo R., et al., 2014, MNRAS, 440, 1498

Seitenzahl I. R., et al., 2013, MNRAS, 429, 1156

Seitenzahl I. R., et al., 2016, A\&A, 592, A57

Shariff H., Dhawan S., Jiao X., Leibundgut B., Trotta R., van Dyk D. A., 2016, MNRAS, 463, 4311

Sim S. A., et al., 2010, ApJ, 714, L52

Stritzinger M., Leibundgut B., Walch S., Contardo G., 2006, A\&A, 450, 241

Stritzinger M. D., et al., 2011, AJ, 142, 156

Suntzeff N. B., 1996, in: Supernovae and supernova remnants, ed. Richard McCray \& Zhenru Wang, Cambridge: Cambridge University Press, 41

Suntzeff, N. B. 2003, in: From Twilight to Highlight, The Physics of Supernovae, ed. W. Hillebrandt \& B. Leibundgut, Heidelberg:Springer, 183

Swartz D. A., Sutherland P. G., Harkness R. P., 1995, ApJ, 446, 766

Taubenberger S., 2017, Handbook of Supernovae, 317, hsn..book

Wygoda N., Elbaz Y., Katz B., 2017, ArXiv e-prints, arXiv:1711.00969

\section{APPENDIX A: TABLES}

Here we present the tables with the data used to produced the plots in this paper. 
Table A1. The parameters for the CfA SNe in the r-band.

\begin{tabular}{lcccccccccc} 
Name & $t_{r_{2}}$ & $\sigma_{t_{r_{2}}}$ & $n_{\text {points }}$ & sbv & $\sigma_{s b v}$ & Wang & Branch & $\overline{\mathcal{F}}_{r_{2}}$ & $\sigma_{\overline{\mathcal{F}}_{r_{2}}}^{\text {lower }}$ & $\sigma_{\overline{\mathcal{F}}_{r_{2}}}^{\text {upper }}$ \\
\hline & & & & & & & & & & \\
SN2001ep & 24.4 & 0.21 & 5 & 0.89 & 0.04 & $\mathrm{BL}$ & $\mathrm{N}$ & 0.486 & 0.44 & 0.53 \\
SN2005am & 15.3 & 0.29 & 11 & 0.70 & 0.02 & $\mathrm{BL}$ & $\mathrm{N}$ & 0.372 & 0.33 & 0.41 \\
SN2005eq & 22.4 & 2.33 & 15 & 1.14 & 0.03 & $\mathrm{SS}$ & $91 \mathrm{~T}$ & 0.527 & 0.50 & 0.56 \\
SN2005hk & 37.1 & 6.61 & 5 & 0.87 & 0.04 & $\mathrm{SS}$ & - & 0.532 & 0.47 & 0.59 \\
SN2006D & 17.3 & 2.38 & 8 & 0.82 & 0.01 & $\mathrm{CN}$ & $\mathrm{N}$ & 0.431 & 0.40 & 0.46 \\
SN2006N & 19.3 & 0.24 & 10 & 0.77 & 0.02 & $\mathrm{CN}$ & $\mathrm{N}$ & 0.421 & 0.39 & 0.45 \\
SN2006S & 24.3 & 1.85 & 11 & 1.12 & 0.07 & $\mathrm{SS}$ & $\mathrm{N}$ & 0.553 & 0.52 & 0.59 \\
SN2006X & 17.0 & 1.04 & 12 & 0.96 & 0.01 & $\mathrm{BL}$ & $\mathrm{HV}$ & 0.464 & 0.42 & 0.51 \\
SN2006ac & 16.8 & 0.36 & 8 & 0.87 & 0.01 & $\mathrm{BL}$ & $\mathrm{HV}$ & 0.423 & 0.39 & 0.46 \\
SN2006az & 16.4 & 6.75 & 18 & 0.84 & 0.01 & $\mathrm{CN}$ & $\mathrm{N}$ & 0.413 & 0.37 & 0.45 \\
SN2006le & 20.4 & 0.3 & 12 & 1.20 & 0.01 & $\mathrm{CN}$ & $\mathrm{N}$ & 0.496 & 0.46 & 0.54 \\
SN2007co & 17.8 & 1.76 & 11 & 0.95 & 0.01 & $\mathrm{BL}$ & $\mathrm{N}$ & 0.473 & 0.39 & 0.56 \\
SN2007kk & 23.3 & 1.65 & 13 & 1.16 & 0.04 & $\mathrm{BL}$ & $\mathrm{N}$ & 0.563 & 0.52 & 0.60 \\
SN2007le & 20.7 & 1.38 & 7 & 1.00 & 0.01 & $\mathrm{BL}$ & $\mathrm{HV}$ & 0.457 & 0.41 & 0.50 \\
SN2007nq & 14.0 & 4.89 & 6 & 0.76 & 0.03 & $\mathrm{BL}$ & $\mathrm{N}$ & 0.346 & 0.28 & 0.42 \\
SN2008Z & 21.1 & 1.69 & 8 & 1.11 & 0.03 & $\mathrm{SS}$ & $\mathrm{N}$ & 0.483 & 0.45 & 0.52 \\
SN2008ae & 21.1 & 7.53 & 9 & 0.84 & 0.07 & $\mathrm{SS}$ & - & 0.478 & 0.41 & 0.55 \\
SN2008ar & 21.9 & 3.09 & 5 & 0.99 & 0.01 & $\mathrm{CN}$ & $\mathrm{N}$ & 0.511 & 0.45 & 0.58 \\
SN2008bf & 22.0 & 0.6 & 9 & 1.03 & 0.02 & $\mathrm{CN}$ & $\mathrm{N}$ & 0.468 & 0.42 & 0.52 \\
& & & & & & & & &
\end{tabular}


Table A2: The parameters for the CSP-I SNe in the r-band.

\begin{tabular}{|c|c|c|c|c|c|c|c|c|c|c|c|c|c|}
\hline Name & $t_{r_{2}}$ & $\sigma_{t_{r_{2}}}$ & $n_{\text {points }}$ & sbv & $\sigma_{s b v}$ & SNID & Wang & Branch & $z_{\text {Helio }}$ & $T_{B(\max )}$ & $\overline{\mathcal{F}}_{r_{2}}$ & $\sigma_{\overline{\mathcal{F}}_{r_{2}}}^{\text {lower }}$ & $\sigma_{\overline{\mathcal{F}}_{r_{2}}}^{\text {upper }}$ \\
\hline SN2004dt & & & & & & & & & & & 0.582 & 0.56 & 0.60 \\
\hline SN2004ef & 15.5 & 0.39 & 16 & 0.81 & 0.00 & Normal & HV & BL & 0.03099 & 53264.0 & 0.386 & 0.35 & 0.42 \\
\hline SN2004eo & 17.9 & 1.53 & 16 & 0.82 & 0.01 & Normal & $\mathrm{N}$ & CL & 0.0157 & 53278.2 & 0.417 & 0.37 & 0.46 \\
\hline SN2004ey & 20.6 & 0.2 & 11 & 1.01 & 0.00 & Normal & $\mathrm{N}$ & $\mathrm{CN}$ & 0.01579 & 53303.9 & 0.439 & 0.40 & 0.48 \\
\hline SN2004gc & 16.2 & 0.93 & 11 & 0.92 & 0.02 & Normal & $\ldots$ & & 0.0307 & 53324.6 & 0.531 & 0.50 & 0.56 \\
\hline SN2004gs & 16.4 & 5.32 & 17 & 0.69 & 0.00 & Normal & $\mathrm{N}$ & CL & 0.02665 & 53356.0 & 0.377 & 0.35 & 0.40 \\
\hline SN2005A & 18.2 & 0.76 & 18 & 0.96 & 0.01 & Normal & $\mathrm{HV}$ & BL & 0.01914 & 53380.4 & 0.47 & 0.45 & 0.49 \\
\hline SN2005M & 18.4 & 0.54 & 17 & 1.21 & 0.00 & $91 \mathrm{~T}$ & $91 \mathrm{~T}$ & SS & 0.02462 & 53405.4 & 0.541 & 0.51 & 0.57 \\
\hline SN2005W & 16.6 & 1.57 & 3 & 0.92 & 0.01 & Normal & $\mathrm{BL}$ & $\ldots$ & 0.00889 & 53411.8 & & & \\
\hline SN2005ag & 18.1 & 4.5 & 12 & 1.08 & 0.01 & Normal & $\mathrm{N}$ & $\mathrm{BL}$ & 0.0798 & 53414.0 & 0.468 & 0.43 & 0.50 \\
\hline SN2005al & 18.9 & 1.69 & 18 & 0.86 & 0.00 & Normal & $\ldots$ & & 0.0124 & 53429.9 & 0.375 & 0.35 & 0.40 \\
\hline SN2005am & 14.6 & 0.27 & 21 & 0.73 & 0.00 & Normal & $\mathrm{HV}$ & $\mathrm{BL}$ & 0.0079 & 53436.6 & 0.346 & 0.33 & 0.36 \\
\hline SN2005be & 19.7 & 0.5 & 10 & 0.75 & 0.02 & Normal & $\ldots$ & & 0.03502 & 53460.6 & & & \\
\hline SN2005bg & 21.9 & 0.38 & 5 & 1.00 & 0.03 & Normal & $\mathrm{N}$ & SS & 0.02309 & 53470.7 & 0.425 & 0.35 & 0.50 \\
\hline SN2005bo & nan & nan & 1 & 0.85 & 0.01 & Normal & $\mathrm{N}$ & $\mathrm{CN}$ & 0.0139 & 53479.8 & & & \\
\hline SN2005el & 16.2 & 0.27 & 9 & 0.83 & 0.00 & Normal & $\mathrm{N}$ & $\mathrm{CN}$ & 0.01491 & 53647.1 & 0.374 & 0.35 & 0.40 \\
\hline SN2005eq & 28.5 & 3.8 & 7 & 1.24 & 0.01 & $91 \mathrm{~T}$ & $91 \mathrm{~T}$ & SS & 0.02898 & 53654.4 & 0.502 & 0.47 & 0.53 \\
\hline SN2005hc & 21.8 & 1.36 & 7 & 1.19 & 0.01 & Normal & $\mathrm{N}$ & $\mathrm{CN}$ & 0.04594 & 53667.1 & 0.526 & 0.49 & 0.56 \\
\hline SN2005hj & & & & & & & & & & & 0.524 & 0.48 & 0.57 \\
\hline SN2005iq & 17.3 & 0.1 & 5 & 0.87 & 0.00 & Normal & $\ldots$ & $\cdots$ & 0.03404 & 53687.7 & 0.374 & 0.33 & 0.42 \\
\hline SN2005kc & 20.6 & 1.01 & 3 & 0.90 & 0.01 & Normal & $\mathrm{N}$ & $\mathrm{CN}$ & 0.01512 & 53697.7 & & & \\
\hline SN2005ke & 17.4 & 9.1 & 9 & 0.42 & 0.00 & $91 \mathrm{bg}$ & $91 \mathrm{bg}$ & CL & 0.00488 & 53698.3 & 0.281 & 0.25 & 0.31 \\
\hline SN2005ki & 15.8 & 0.41 & 9 & 0.82 & 0.00 & Normal & $\mathrm{N}$ & $\mathrm{CN}$ & 0.01921 & 53705.6 & 0.349 & 0.31 & 0.38 \\
\hline SN2005ku & nan & 5.51 & 3 & 1.19 & 0.04 & Normal & $\mathrm{HV}$ & $\mathrm{CN}$ & 0.04544 & 53698.4 & & & \\
\hline SN2005lu & 24.0 & 0.77 & 6 & 1.13 & 0.03 & Normal & $\ldots$ & $\ldots$ & 0.03201 & 53711.9 & & & \\
\hline $\mathrm{SN} 2005 \mathrm{mc}$ & & & & & & & & & & & 0.388 & 0.35 & 0.42 \\
\hline SN2005na & & & & & & & & & & & 0.447 & 0.42 & 0.47 \\
\hline SN2006D & 14.2 & 0.11 & 12 & 0.81 & 0.00 & Normal & $\mathrm{N}$ & $\mathrm{CN}$ & 0.00853 & 53757.3 & 0.346 & 0.32 & 0.37 \\
\hline SN2006X & 19.5 & 0.93 & 9 & 0.97 & 0.01 & Normal & $\mathrm{HV}$ & $\mathrm{BL}$ & 0.00524 & 53785.8 & 0.456 & 0.42 & 0.49 \\
\hline SN2006ax & 20.9 & 3.28 & 7 & 0.98 & 0.00 & Normal & $\mathrm{N}$ & $\mathrm{CN}$ & 0.01674 & 53827.1 & 0.44 & 0.40 & 0.48 \\
\hline SN2006bh & 16.8 & 0.45 & 10 & 0.80 & 0.00 & Normal & $\ldots$ & & 0.01085 & 53833.4 & 0.343 & 0.32 & 0.37 \\
\hline SN2006br & 22.7 & 0.84 & 7 & 0.91 & 0.03 & Normal & $\mathrm{HV}$ & BL & 0.02459 & 53851.2 & & & \\
\hline SN2006bt & & & & & & & & & & & 0.533 & 0.49 & 0.57 \\
\hline SN2006ef & 37.0 & 0.26 & 5 & 0.84 & 0.02 & Normal & $\mathrm{HV}$ & BL & 0.01788 & 53969.7 & 0.551 & 0.51 & 0.59 \\
\hline SN2006ej & 18.8 & 0.1 & 5 & 0.82 & 0.02 & Normal & $\mathrm{HV}$ & $\mathrm{BL}$ & 0.02045 & 53975.6 & & & \\
\hline SN2006eq & nan & 7.06 & 9 & 0.62 & 0.03 & Normal & $\mathrm{N}$ & CL & 0.0495 & 53977.1 & & & \\
\hline SN2006et & & & & & & & & & & & 0.509 & 0.47 & 0.55 \\
\hline SN2006ev & 13.6 & 1.34 & 7 & 0.84 & 0.02 & Normal & $\ldots$ & & 0.02873 & 53989.1 & & & \\
\hline SN2006gj & 35.5 & 4.59 & 5 & 0.66 & 0.01 & Normal & $\mathrm{N}$ & CL & 0.02835 & 53999.6 & 0.369 & 0.32 & 0.41 \\
\hline SN2006gt & & & & & & & & & & & 0.432 & 0.38 & 0.48 \\
\hline SN2006hb & 37.8 & 9.55 & 7 & 0.66 & 0.00 & $86 \mathrm{G}$ & $91 \mathrm{bg}$ & & 0.01534 & 53998.7 & & & \\
\hline SN2006hx & 17.1 & 0.55 & 4 & 0.90 & 0.02 & Normal & $\mathrm{N}$ & SS & 0.04549 & 54022.1 & & & \\
\hline SN2006is & 22.4 & 0.62 & 5 & 1.14 & 0.03 & Normal & $\mathrm{HV}$ & $\mathrm{CN}$ & 0.0314 & 54008.4 & & & \\
\hline SN2006kf & 14.7 & 0.29 & 7 & 0.73 & 0.00 & Normal & $\mathrm{N}$ & CL & 0.0213 & 54041.3 & 0.345 & 0.32 & 0.37 \\
\hline SN2006lu & 25.9 & 1.21 & 6 & 1.05 & 0.03 & Normal & $\ldots$ & $\ldots$ & 0.0534 & 54034.1 & & & \\
\hline SN2006mr & 19.7 & 7.67 & 9 & 0.26 & 0.00 & $91 \mathrm{bg}$ & $91 \mathrm{bg}$ & CL & 0.00587 & 54050.1 & 0.253 & 0.22 & 0.29 \\
\hline SN2006ob & 15.2 & 0.39 & 5 & 0.74 & 0.01 & Normal & $\ldots$ & $\ldots$ & 0.05924 & 54063.4 & 0.359 & 0.31 & 0.41 \\
\hline SN2006os & 18.5 & 0.54 & 6 & 0.94 & 0.02 & Normal & $\mathrm{N}$ & CL & 0.03281 & 54062.8 & & & \\
\hline SN2006ot & & & & & & & & & & & 0.618 & 0.57 & 0.66 \\
\hline SN2006py & 19.0 & 0.09 & 2 & 0.95 & 0.03 & Normal & $\cdots$ & & 0.0579 & 54070.6 & & & \\
\hline SN2007A & 19.6 & 0.08 & 2 & 1.00 & 0.01 & Normal & $\mathrm{N}$ & $\mathrm{CN}$ & 0.01765 & 54112.8 & & & \\
\hline SN2007N & 38.4 & 2.91 & 6 & 0.30 & 0.01 & $91 \mathrm{bg}$ & $91 \mathrm{bg}$ & CL & 0.01288 & 54123.8 & 0.26 & 0.22 & 0.30 \\
\hline SN2007S & 21.8 & 0.94 & 7 & 1.12 & 0.01 & $91 \mathrm{~T}$ & $91 \mathrm{~T}$ & SS & 0.01388 & 54144.6 & 0.514 & 0.47 & 0.56 \\
\hline SN2007af & 18.5 & 0.4 & 9 & 0.93 & 0.00 & Normal & $\mathrm{N}$ & $\mathrm{BL}$ & 0.00546 & 54174.3 & 0.436 & 0.40 & 0.47 \\
\hline SN2007ai & 22.7 & 1.56 & 6 & 1.25 & 0.01 & $91 \mathrm{~T}$ & $91 \mathrm{~T}$ & SS & 0.03166 & 54171.8 & 0.594 & 0.55 & 0.64 \\
\hline SN2007as & 18.9 & 0.62 & 6 & 0.88 & 0.00 & Normal & $\mathrm{HV}$ & $\mathrm{BL}$ & 0.01757 & 54181.3 & 0.407 & 0.38 & 0.44 \\
\hline SN2007ax & 33.8 & 0.41 & 4 & 0.36 & 0.01 & $91 \mathrm{bg}$ & $91 \mathrm{bg}$ & CL & 0.00686 & 54187.6 & & & \\
\hline
\end{tabular}


Characterising the secondary maximum in the r-band for Type Ia Supernovae

\begin{tabular}{|c|c|c|c|c|c|c|c|c|c|c|c|c|c|}
\hline SN2007ba & & & & & & & & & & & 0.368 & 0.30 & 0.43 \\
\hline SN2007bc & 24.6 & 0.12 & 5 & 0.89 & 0.01 & Normal & $\mathrm{N}$ & $\mathrm{CL}$ & 0.02077 & 54200.1 & 0.437 & 0.38 & 0.49 \\
\hline SN2007bd & & & & & & & & & & & 0.369 & 0.31 & 0.42 \\
\hline SN2007bm & 18.3 & 0.79 & 5 & 0.91 & 0.01 & Normal & $\mathrm{N}$ & $\mathrm{CN}$ & 0.00621 & 54224.6 & 0.425 & 0.39 & 0.46 \\
\hline SN2007jg & 20.0 & 0.84 & 8 & 0.92 & 0.01 & Normal & $\mathrm{HV}$ & $\mathrm{BL}$ & 0.03713 & 54367.2 & 0.417 & 0.38 & 0.45 \\
\hline SN2007jh & 33.4 & 5.59 & 5 & 0.59 & 0.01 & $86 \mathrm{G}$ & $91 \mathrm{bg}$ & $\ldots$ & 0.0408 & 54365.3 & 0.349 & 0.29 & 0.41 \\
\hline SN2007le & 19.3 & 0.74 & 6 & 1.02 & 0.00 & Normal & $\mathrm{HV}$ & BL & 0.00672 & 54398.7 & 0.452 & 0.41 & 0.49 \\
\hline SN2007nq & 13.6 & 0.12 & 7 & 0.75 & 0.01 & Normal & $\mathrm{HV}$ & BL & 0.04503 & 54398.2 & 0.355 & 0.32 & 0.39 \\
\hline SN2007on & 13.2 & 8.2 & 11 & 0.57 & 0.00 & Normal & $\mathrm{N}$ & CL & 0.00649 & 54419.7 & 0.299 & 0.27 & 0.33 \\
\hline SN2008C & 19.9 & 0.27 & 6 & 0.95 & 0.02 & Normal & $\mathrm{N}$ & SS & 0.01662 & 54466.3 & 0.465 & 0.43 & 0.50 \\
\hline SN2008R & 15.7 & 0.07 & 8 & 0.60 & 0.01 & $91 \mathrm{bg}$ & $91 \mathrm{bg}$ & CL & 0.0135 & 54494.3 & 0.326 & 0.30 & 0.36 \\
\hline SN2008bc & 18.2 & 2.59 & 11 & 1.03 & 0.00 & Normal & $\mathrm{N}$ & $\mathrm{CN}$ & 0.01509 & 54548.7 & 0.446 & 0.41 & 0.48 \\
\hline SN2008bq & 22.4 & 0.33 & 6 & 1.15 & 0.01 & Normal & $\mathrm{N}$ & $\mathrm{CN}$ & 0.034 & 54562.9 & 0.499 & 0.46 & 0.53 \\
\hline SN2008fp & 17.6 & 2.32 & 10 & 1.05 & 0.01 & Normal & $\mathrm{N}$ & $\mathrm{CN}$ & 0.00566 & 54729.7 & 0.455 & 0.43 & 0.48 \\
\hline SN2008gp & 22.8 & 3.28 & 10 & 0.97 & 0.01 & Normal & $\ldots$ & $\ldots$ & 0.03341 & 54778.6 & 0.453 & 0.39 & 0.51 \\
\hline SN2008hv & 16.4 & 1.79 & 11 & 0.85 & 0.00 & Normal & $\mathrm{N}$ & $\mathrm{CN}$ & 0.01255 & 54816.8 & 0.353 & 0.30 & 0.40 \\
\hline SN2008ia & & & & & & & & & & & 0.377 & 0.34 & 0.41 \\
\hline SN2009F & 14.0 & 0.05 & 7 & 0.34 & 0.01 & $91 \mathrm{bg}$ & $91 \mathrm{bg}$ & CL & 0.01296 & 54841.8 & 0.24 & 0.19 & 0.29 \\
\hline SN2009dc & & & & & & & & & & & 0.666 & 0.61 & 0.72 \\
\hline
\end{tabular}


Table A3: The parameters for the CfA SNe in the i-band.

\begin{tabular}{|c|c|c|c|c|c|c|}
\hline Name & $\overline{\mathcal{F}}_{i_{2}}$ & $\sigma_{\overline{\mathcal{F}}_{i_{2}}}^{\text {lower }}$ & $\sigma_{\overline{\mathcal{F}}_{i_{2}}}^{\text {upper }}$ & $t_{i_{2}}$ & $\sigma_{t_{i_{2}}}$ & $n_{\text {points }}$ \\
\hline SN2001V & 0.726 & 0.63 & 0.82 & 28.8 & 0.41 & 7 \\
\hline SN2001ep & & & & 18.3 & 1.11 & 4 \\
\hline SN2002bo & 0.654 & 0.55 & 0.76 & 23.3 & 0.37 & 6 \\
\hline SN2002cr & & & & 24.8 & 0.54 & 4 \\
\hline SN2002fk & 0.54 & 0.50 & 0.58 & 26.2 & 0.48 & 6 \\
\hline SN2002ha & 0.489 & 0.40 & 0.57 & 23.7 & 0.29 & 4 \\
\hline SN2002hu & & & & 27.8 & 0.9 & 4 \\
\hline SN2003W & 0.691 & 0.63 & 0.75 & 25.4 & 0.35 & 5 \\
\hline SN2003cg & 0.62 & 0.57 & 0.67 & 24.4 & 0.24 & 5 \\
\hline SN2003du & & & & 28.6 & 0.61 & 4 \\
\hline SN2003kf & 0.515 & 0.48 & 0.55 & 27.4 & 0.61 & 5 \\
\hline SN2005am & 0.489 & 0.45 & 0.53 & 18.5 & 0.12 & 10 \\
\hline SN2005el & 0.438 & 0.42 & 0.46 & 22.2 & 0.15 & 23 \\
\hline SN2005eq & 0.556 & 0.52 & 0.60 & 31.5 & 0.81 & 15 \\
\hline SN2005hc & 0.552 & 0.49 & 0.62 & 30.1 & 1.07 & 10 \\
\hline SN2005hk & 0.653 & 0.59 & 0.71 & nan & 1.38 & 5 \\
\hline SN2005kc & 0.529 & 0.44 & 0.62 & 22.7 & 0.54 & 6 \\
\hline SN2005ke & 0.467 & 0.39 & 0.54 & nan & 8.61 & 7 \\
\hline SN2005ki & & & & 16.2 & 2.24 & 5 \\
\hline SN2005lz & 0.504 & 0.43 & 0.58 & 25.2 & 0.35 & 5 \\
\hline $\mathrm{SN} 2005 \mathrm{mc}$ & & & & 31.4 & 8.81 & 4 \\
\hline SN2005ms & 0.501 & 0.46 & 0.55 & 26.4 & 0.59 & 5 \\
\hline SN2005mz & 0.351 & 0.28 & 0.42 & 33.8 & 1.05 & 7 \\
\hline SN2006D & 0.399 & 0.37 & 0.43 & 22.4 & 1.4 & 8 \\
\hline SN2006N & 0.555 & 0.52 & 0.59 & 20.7 & 0.39 & 7 \\
\hline SN2006S & 0.571 & 0.53 & 0.61 & 30.7 & 0.3 & 12 \\
\hline SN2006X & 0.591 & 0.54 & 0.64 & 25.7 & 0.38 & 14 \\
\hline SN2006ac & 0.452 & 0.41 & 0.49 & 24.1 & 0.33 & 9 \\
\hline SN2006ax & 0.515 & 0.46 & 0.57 & & & \\
\hline SN2006az & 0.502 & 0.46 & 0.54 & 22.3 & 1.35 & 18 \\
\hline SN2006bt & 0.678 & 0.63 & 0.73 & & & \\
\hline SN2006cc & 0.511 & 0.44 & 0.59 & 29.5 & 0.33 & 6 \\
\hline SN2006gj & 0.529 & 0.46 & 0.60 & 19.0 & 0.49 & 5 \\
\hline SN2006le & 0.542 & 0.49 & 0.59 & 30.8 & 2.96 & 12 \\
\hline SN2006lf & 0.413 & 0.35 & 0.47 & 20.5 & 0.32 & 8 \\
\hline SN2006ob & 0.41 & 0.32 & 0.49 & 17.3 & 0.29 & 7 \\
\hline SN2006ou & & & & nan & 3.02 & 4 \\
\hline SN2007aj & 0.856 & 0.79 & 0.92 & 24.6 & 0.46 & 10 \\
\hline SN2007bj & 0.829 & 0.77 & 0.89 & 23.8 & 0.27 & 10 \\
\hline SN2007cb & & & & 19.5 & 1.88 & 5 \\
\hline SN2007cf & & & & nan & 3.3 & 6 \\
\hline SN2007cn & & & & nan & nan & 5 \\
\hline SN2007co & 0.591 & 0.50 & 0.68 & 26.3 & 0.34 & 11 \\
\hline SN2007hg & 1.042 & 0.91 & 1.17 & nan & 2.25 & 10 \\
\hline SN2007hj & 0.462 & 0.42 & 0.50 & 15.9 & 0.32 & 9 \\
\hline SN2007hu & & & & 30.1 & 0.89 & 4 \\
\hline SN2007if & & & & nan & 3.31 & 6 \\
\hline SN2007ir & 0.759 & 0.57 & 0.95 & nan & 3.93 & 4 \\
\hline SN2007jg & 0.52 & 0.46 & 0.58 & 27.2 & 0.56 & 9 \\
\hline SN2007kk & 0.7 & 0.65 & 0.75 & 27.5 & 0.49 & 13 \\
\hline SN2007le & 0.589 & 0.53 & 0.65 & 27.3 & 0.19 & 7 \\
\hline SN2007nq & 0.46 & 0.39 & 0.53 & 15.8 & 0.52 & 7 \\
\hline SN2007ob & 0.613 & 0.53 & 0.70 & 28.1 & 0.76 & 6 \\
\hline SN2007ss & 0.546 & 0.50 & 0.60 & 24.1 & 0.43 & 6 \\
\hline SN2007sw & 0.634 & 0.59 & 0.68 & 26.2 & 0.4 & 11 \\
\hline SN2007ux & 0.454 & 0.40 & 0.51 & & & \\
\hline SN2008C & 0.584 & 0.53 & 0.64 & 24.0 & 0.51 & 5 \\
\hline
\end{tabular}




\begin{tabular}{|c|c|c|c|c|c|c|}
\hline SN2008Y & & & & 28.3 & 1.58 & 6 \\
\hline SN2008Z & 0.468 & 0.43 & 0.51 & 30.6 & 0.27 & 10 \\
\hline SN2008ac & & & & 26.0 & 2.32 & 3 \\
\hline SN2008ae & 0.622 & 0.54 & 0.71 & 30.1 & 1.87 & 8 \\
\hline SN2008ar & 0.622 & 0.56 & 0.68 & 23.1 & 0.6 & 5 \\
\hline SN2008at & 0.707 & 0.63 & 0.78 & & & \\
\hline SN2008bf & 0.501 & 0.44 & 0.56 & 28.1 & 0.82 & 9 \\
\hline SN2008bi & & & & 38.7 & 0.91 & 6 \\
\hline SN2008bw & 0.739 & 0.64 & 0.84 & nan & nan & 5 \\
\hline SN2008cd & & & & 14.2 & 1.95 & 6 \\
\hline $\mathrm{SN} 2008 \mathrm{~cm}$ & 0.485 & 0.40 & 0.57 & 22.0 & 1.54 & 7 \\
\hline SN2008fr & 0.63 & 0.58 & 0.68 & 29.5 & 0.76 & 10 \\
\hline SN2008gb & 0.546 & 0.47 & 0.62 & 23.8 & 1.28 & 6 \\
\hline SN2008gl & 0.513 & 0.45 & 0.58 & 22.1 & 0.5 & 5 \\
\hline SN2008hm & 0.573 & 0.52 & 0.63 & 24.4 & 0.67 & 10 \\
\hline SN2008hs & 0.628 & 0.56 & 0.70 & 16.4 & 0.26 & 8 \\
\hline SN2008hv & & & & 19.5 & 0.77 & 4 \\
\hline SN2009D & 0.592 & 0.54 & 0.64 & 22.9 & 0.17 & 7 \\
\hline SN2009Y & 0.555 & 0.52 & 0.59 & 28.7 & 0.41 & 12 \\
\hline SN2009ad & 0.558 & 0.50 & 0.62 & 28.0 & 0.32 & 9 \\
\hline SN2009al & 0.632 & 0.58 & 0.68 & 22.0 & 0.45 & 8 \\
\hline SN2009an & 0.489 & 0.43 & 0.55 & 22.2 & 0.39 & 8 \\
\hline SN2009bv & 0.432 & 0.36 & 0.50 & 16.2 & 5.09 & 6 \\
\hline SN2009dc & 0.835 & 0.80 & 0.87 & 20.4 & 1.41 & 14 \\
\hline SN2009do & 0.631 & 0.56 & 0.70 & 23.4 & 0.76 & 8 \\
\hline SN2009ds & 0.452 & 0.37 & 0.54 & 30.8 & 0.46 & 5 \\
\hline SN2009ig & 0.501 & 0.47 & 0.53 & 30.0 & 0.31 & 9 \\
\hline SN2009jr & 0.513 & 0.43 & 0.59 & 31.2 & 0.37 & 8 \\
\hline SN2009kk & 0.529 & 0.49 & 0.57 & 23.5 & 0.49 & 7 \\
\hline SN2009kq & 0.564 & 0.52 & 0.60 & 27.0 & 0.23 & 5 \\
\hline SN2009lf & 0.601 & 0.53 & 0.67 & 23.3 & 0.55 & 6 \\
\hline SN2010Y & 0.421 & 0.34 & 0.50 & 16.6 & 0.31 & 8 \\
\hline SN2010ag & 0.577 & 0.52 & 0.63 & 27.7 & 0.98 & 6 \\
\hline SN2010ai & 0.42 & 0.27 & 0.57 & 22.8 & 3.5 & 6 \\
\hline
\end{tabular}


14 Papadogiannakis S. et al.

Table A4: The parameters for the CSP-I SNe in the i-band.

\begin{tabular}{|c|c|c|c|c|c|c|}
\hline Name & $\overline{\mathcal{F}}_{i_{2}}$ & $\sigma_{\overline{\mathcal{F}}_{i_{2}}}^{\text {lower }}$ & $\sigma_{\overline{\mathcal{F}}_{i_{2}}}^{\text {upper }}$ & $t_{i_{2}}$ & $\sigma_{t_{i_{2}}}$ & $n_{\text {points }}$ \\
\hline SN2004dt & 0.759 & 18.322 & 19.63 & & & \\
\hline SN2004ef & 0.5 & 11.463 & 13.531 & 22.6 & 0.2 & 16 \\
\hline SN2004eo & 0.548 & 12.364 & 15.027 & 19.1 & 0.91 & 16 \\
\hline SN2004ey & 0.518 & 11.854 & 14.032 & 28.2 & 0.14 & 11 \\
\hline SN2004gc & 0.679 & 16.01 & 17.915 & 19.5 & 1.21 & 10 \\
\hline SN2004gs & 0.458 & 10.732 & 12.148 & 18.5 & 0.46 & 16 \\
\hline SN2005A & 0.693 & 16.33 & 18.308 & 25.1 & 0.18 & 18 \\
\hline SN2005M & 0.568 & 13.313 & 15.09 & 25.9 & 1.46 & 17 \\
\hline SN2005ag & 0.569 & 12.974 & 15.485 & 30.2 & 4.72 & 12 \\
\hline SN2005al & 0.493 & 11.507 & 13.141 & 23.8 & 0.14 & 18 \\
\hline SN2005am & 0.487 & 11.474 & 12.888 & 19.1 & 0.14 & 19 \\
\hline SN2005be & 0.737 & 16.616 & 20.245 & 25.1 & 0.25 & 10 \\
\hline SN2005bg & 0.52 & 10.126 & 15.869 & 23.9 & 0.66 & 5 \\
\hline SN2005el & 0.432 & 9.877 & 11.711 & 21.9 & 0.11 & 9 \\
\hline SN2005eq & 0.524 & 12.064 & 14.148 & 32.0 & 0.26 & 7 \\
\hline SN2005hc & 0.563 & 12.944 & 15.224 & 31.7 & 0.21 & 7 \\
\hline SN2005hj & 0.583 & 13.268 & 15.878 & 32.7 & 0.11 & 5 \\
\hline SN2005iq & 0.398 & 8.525 & 11.366 & 24.4 & 0.23 & 5 \\
\hline SN2005ir & & & & 26.5 & 0.3 & 4 \\
\hline SN2005kc & & & & 22.6 & 0.16 & 4 \\
\hline SN2005ke & 0.369 & 8.38 & 10.055 & 17.1 & 6.87 & 9 \\
\hline SN2005ki & 0.426 & 9.605 & 11.688 & 21.6 & 0.14 & 9 \\
\hline SN2005ku & & & & 28.3 & 1.12 & 3 \\
\hline SN2005lu & 0.779 & 18.17 & 20.775 & 30.7 & 0.34 & 6 \\
\hline SN2005mc & 0.51 & 11.792 & 13.7 & 17.8 & 0.23 & 11 \\
\hline SN2005na & 0.547 & 12.67 & 14.682 & 26.1 & 0.37 & 11 \\
\hline SN2006D & 0.381 & 8.949 & 10.078 & 22.8 & 0.2 & 12 \\
\hline SN2006X & 0.598 & 13.929 & 15.947 & 24.7 & 0.13 & 9 \\
\hline SN2006ax & 0.497 & 11.151 & 13.686 & & & \\
\hline SN2006bd & & & & nan & 0.06 & 5 \\
\hline SN2006bh & 0.422 & 9.787 & 11.33 & 21.2 & 0.1 & 10 \\
\hline SN2006br & 0.696 & 15.594 & 19.196 & 26.6 & 1.35 & 7 \\
\hline SN2006bt & 0.668 & 15.846 & 17.543 & & & \\
\hline SN2006ef & 0.786 & 18.396 & 20.916 & 18.5 & 0.15 & 5 \\
\hline SN2006ej & 0.573 & 13.322 & 15.322 & 23.5 & 0.18 & 5 \\
\hline SN2006eq & 0.531 & 12.069 & 14.474 & 18.1 & 0.58 & 9 \\
\hline SN2006et & 0.584 & 13.672 & 15.529 & & & \\
\hline SN2006ev & 0.488 & 10.689 & 13.719 & 19.5 & 0.26 & 7 \\
\hline SN2006gj & 0.414 & 9.132 & 11.583 & 13.4 & 6.65 & 5 \\
\hline SN2006gt & 0.519 & 11.658 & 14.27 & & & \\
\hline SN2006hb & 0.733 & 17.354 & 19.304 & 18.8 & 4.21 & 7 \\
\hline SN2006is & 0.622 & 14.411 & 16.67 & 32.1 & 0.18 & 5 \\
\hline SN2006kf & 0.405 & 9.347 & 10.902 & 18.2 & 0.18 & 7 \\
\hline SN2006lu & 0.657 & 15.086 & 17.751 & 27.9 & 0.91 & 6 \\
\hline SN2006mr & 0.324 & 7.119 & 9.099 & 19.3 & 0.14 & 9 \\
\hline SN2006ob & 0.469 & 10.28 & 13.192 & 18.8 & 0.42 & 5 \\
\hline SN2006os & 0.605 & 13.954 & 16.272 & 23.4 & 0.37 & 6 \\
\hline SN2006ot & 0.723 & 16.846 & 19.293 & & & \\
\hline SN2007N & 0.352 & 7.6 & 10.0 & 34.4 & 5.82 & 6 \\
\hline SN2007S & 0.576 & 13.206 & 15.593 & 30.2 & 0.17 & 7 \\
\hline SN2007af & 0.512 & 11.816 & 13.795 & 25.5 & 0.33 & 9 \\
\hline SN2007ai & 0.626 & 14.482 & 16.833 & 30.9 & 0.85 & 6 \\
\hline SN2007as & 0.511 & 11.552 & 14.016 & 24.8 & 0.13 & 6 \\
\hline SN2007ba & 0.48 & 10.313 & 13.691 & 15.0 & 2.88 & 7 \\
\hline SN2007bc & & & & 23.2 & 3.56 & 5 \\
\hline SN2007bd & 0.484 & 10.464 & 13.735 & 22.8 & 0.17 & 5 \\
\hline SN2007bm & 0.584 & 13.27 & 15.922 & 22.5 & 0.15 & 5 \\
\hline
\end{tabular}




\begin{tabular}{lcccccc} 
SN2007ca & & & & 29.5 & 0.32 & 4 \\
SN2007if & & & & 22.5 & 0.68 & 6 \\
SN2007jg & 0.466 & 9.929 & 13.378 & 26.4 & 2.62 & 7 \\
SN2007jh & 0.476 & 10.109 & 13.676 & 14.4 & 4.58 & 5 \\
SN2007le & 0.578 & 13.133 & 15.782 & 27.4 & 0.3 & 6 \\
SN2007nq & 0.468 & 10.742 & 12.675 & 18.9 & 0.19 & 7 \\
SN2007on & 0.406 & 9.221 & 11.072 & 14.5 & 0.25 & 11 \\
SN2008C & 0.66 & 15.328 & 17.678 & 21.0 & 0.31 & 6 \\
SN2008R & 0.444 & 10.25 & 11.936 & 16.4 & 0.13 & 8 \\
SN2008bc & 0.505 & 11.511 & 13.751 & 29.3 & 0.56 & 11 \\
SN2008bq & 0.591 & 13.387 & 16.163 & 30.4 & 1.84 & 6 \\
SN2008fp & 0.518 & 12.181 & 13.709 & 27.7 & 0.17 & 10 \\
SN2008gp & 0.544 & 11.801 & 15.378 & 25.3 & 4.59 & 10 \\
SN2008hv & 0.44 & 9.412 & 12.602 & 22.5 & 0.52 & 11 \\
SN2008ia & 0.488 & 11.149 & 13.261 & 23.2 & 3.31 & 8 \\
SN2009F & 0.296 & 6.165 & 8.653 & 24.7 & 3.14 & 7 \\
SN2009dc & & & & 20.8 & 0.72 & 4 \\
& & & & & & \\
\hline
\end{tabular}


16 Papadogiannakis S. et al.

Table A5. The $t_{0}$ values from the CSP-I SNe with sufficient multi-band data.

\begin{tabular}{ccc} 
Name & $t_{0}$ & $\sigma_{t_{0}}$ \\
\hline & & \\
SN2004eo & 36.6655 & 0.0802 \\
SN2004ey & 34.8975 & 0.0284 \\
SN2005M & 38.2209 & 0.1411 \\
SN2005el & 29.4895 & 0.0517 \\
SN2005ke & 31.3489 & 0.0548 \\
SN2005ki & 30.6891 & 0.0613 \\
SN2006D & 29.8909 & 0.0486 \\
SN2006ax & 35.8279 & 0.0738 \\
SN2006et & 38.3934 & 0.0918 \\
SN2006kf & 28.9012 & 0.0587 \\
SN2006mr & 26.2137 & 0.0234 \\
SN2007S & 40.1609 & 0.0925 \\
SN2007af & 34.4224 & 0.0766 \\
SN2007ax & 27.8355 & 0.0396 \\
SN2007bc & 28.7434 & 0.046 \\
SN2007bd & 31.5881 & 0.0021 \\
SN2007le & 37.5743 & 0.0803 \\
SN2007on & 29.0764 & 0.0589 \\
SN2008bc & 36.7482 & 0.052 \\
SN2008hv & 30.8722 & 0.0686 \\
SN2008ia & 29.1072 & 0.2377 \\
& &
\end{tabular}


Table A6: The parameters for the PTF and iPTF SNe in the r-band.

\begin{tabular}{|c|c|c|c|}
\hline Name & $t_{r_{2}}$ & $\sigma_{t_{r_{2}}}^{\text {lower }}$ & $\sigma_{t_{r_{2}}}^{\text {upper }}$ \\
\hline PTF10rgn & 0.454 & 0.377 & 0.532 \\
\hline iPTF16fmb & 0.251 & 0.113 & 0.389 \\
\hline iPTF13aol & 0.463 & 0.398 & 0.527 \\
\hline iPTF13dhp & 0.528 & 0.435 & 0.621 \\
\hline iPTF16aas & 0.245 & 0.093 & 0.398 \\
\hline PTF12eac & 0.484 & 0.305 & 0.663 \\
\hline iPTF16gdp & 0.338 & 0.24 & 0.437 \\
\hline iPTF13ani & 0.573 & 0.494 & 0.651 \\
\hline PTF12fxn & 0.36 & 0.176 & 0.545 \\
\hline PTF10hpp & 0.501 & 0.4 & 0.601 \\
\hline PTF10tce & 0.557 & 0.473 & 0.64 \\
\hline PTF10hdn & 0.544 & 0.46 & 0.628 \\
\hline iPTF13ax & 0.505 & 0.448 & 0.561 \\
\hline iPTF16eka & 0.418 & 0.351 & 0.484 \\
\hline PTF10hdm & 0.488 & 0.416 & 0.561 \\
\hline PTF11htb & 0.49 & 0.402 & 0.578 \\
\hline iPTF16hun & 0.293 & 0.089 & 0.498 \\
\hline PTF10nyt & 0.522 & 0.412 & 0.631 \\
\hline PTF10kzf & 0.582 & 0.504 & 0.661 \\
\hline PTF11dec & 0.495 & 0.442 & 0.548 \\
\hline PTF12cjg & 0.447 & 0.259 & 0.635 \\
\hline PTF10urn & 0.459 & 0.355 & 0.563 \\
\hline PTF10uzi & 0.214 & 0.168 & 0.26 \\
\hline PTF12gmf & 0.241 & 0.05 & 0.431 \\
\hline iPTF13ez & 0.49 & 0.438 & 0.541 \\
\hline PTF11blu & 0.524 & 0.459 & 0.589 \\
\hline iPTF14yl & 0.688 & 0.586 & 0.791 \\
\hline PTF10rpt & 0.507 & 0.438 & 0.575 \\
\hline PTF12gmq & 0.444 & 0.246 & 0.642 \\
\hline iPTF14aia & 0.406 & 0.331 & 0.482 \\
\hline PTF11dzm & 0.286 & 0.191 & 0.381 \\
\hline iPTF14apu & 0.341 & 0.182 & 0.5 \\
\hline PTF11kml & 0.478 & 0.367 & 0.588 \\
\hline PTF09alu & 0.315 & 0.223 & 0.407 \\
\hline iPTF16dp & 0.41 & 0.31 & 0.51 \\
\hline PTF10tfs & 0.313 & 0.233 & 0.394 \\
\hline PTF10hcu & 0.435 & 0.331 & 0.539 \\
\hline iPTF13apn & 0.481 & 0.427 & 0.536 \\
\hline PTF11bok & 0.501 & 0.421 & 0.58 \\
\hline PTF10pvi & 0.457 & 0.366 & 0.548 \\
\hline PTF11rnu & 0.356 & 0.229 & 0.483 \\
\hline PTF10hne & 0.493 & 0.426 & 0.56 \\
\hline iPTF16grm & 0.348 & 0.255 & 0.441 \\
\hline iPTF14axv & 0.467 & 0.4 & 0.533 \\
\hline PTF11ivb & 0.395 & 0.32 & 0.47 \\
\hline PTF10hrw & 0.449 & 0.368 & 0.53 \\
\hline PTF11cji & 0.48 & 0.367 & 0.592 \\
\hline iPTF13ckk & 0.527 & 0.471 & 0.583 \\
\hline PTF11bas & 0.482 & 0.42 & 0.543 \\
\hline PTF10ucj & 0.424 & 0.308 & 0.539 \\
\hline PTF12mj & 0.543 & 0.465 & 0.621 \\
\hline PTF10nvh & 0.553 & 0.459 & 0.647 \\
\hline PTF11cmg & 0.365 & 0.271 & 0.46 \\
\hline PTF11cyv & 0.482 & 0.364 & 0.6 \\
\hline PTF11cml & 0.445 & 0.367 & 0.524 \\
\hline iPTF13beg & 0.348 & 0.284 & 0.411 \\
\hline PTF10aayx & 0.394 & 0.284 & 0.503 \\
\hline
\end{tabular}




\begin{tabular}{|c|c|c|c|}
\hline iPTF14alb & 0.361 & 0.126 & 0.596 \\
\hline PTF10qrj & 0.337 & 0.27 & 0.404 \\
\hline iPTF13dnj & 0.4 & 0.336 & 0.465 \\
\hline iPTF14amb & 0.482 & 0.383 & 0.58 \\
\hline PTF10qnn & 0.471 & 0.379 & 0.564 \\
\hline iPTF14bcl & 0.467 & 0.42 & 0.513 \\
\hline PTF11xe & 0.553 & 0.425 & 0.681 \\
\hline iPTF13adg & 0.478 & 0.4 & 0.556 \\
\hline iPTF13ai & 0.433 & 0.371 & 0.496 \\
\hline PTF12fuu & 0.405 & 0.162 & 0.649 \\
\hline PTF10aajv & 0.302 & 0.265 & 0.339 \\
\hline PTF12kim & 0.519 & 0.393 & 0.644 \\
\hline PTF09dxo & 0.391 & 0.274 & 0.507 \\
\hline iPTF13ccm & 0.482 & 0.443 & 0.521 \\
\hline PTF10mla & 0.458 & 0.337 & 0.578 \\
\hline PTF12dhb & 0.499 & 0.428 & 0.57 \\
\hline PTF10tum & 0.552 & 0.411 & 0.694 \\
\hline iPTF13s & 0.504 & 0.448 & 0.559 \\
\hline PTF12dhk & 0.611 & 0.543 & 0.68 \\
\hline PTF10ucl & 0.413 & 0.301 & 0.526 \\
\hline PTF10qqw & 0.514 & 0.447 & 0.58 \\
\hline PTF12cnl & 0.531 & 0.455 & 0.607 \\
\hline PTF10qkf & 0.47 & 0.397 & 0.542 \\
\hline PTF12keu & 0.546 & 0.416 & 0.676 \\
\hline PTF10glo & 0.578 & 0.544 & 0.612 \\
\hline PTF11qpc & 0.537 & 0.441 & 0.633 \\
\hline PTF10iah & 0.496 & 0.397 & 0.596 \\
\hline PTF10duy & 0.471 & 0.412 & 0.53 \\
\hline iPTF13dkx & 0.525 & 0.471 & 0.579 \\
\hline PTF10rhi & 0.551 & 0.432 & 0.67 \\
\hline PTF10urj & 0.418 & 0.312 & 0.524 \\
\hline PTF12dgy & 0.381 & 0.302 & 0.459 \\
\hline iPTF13czs & 0.47 & 0.417 & 0.523 \\
\hline iPTF13dkl & 0.476 & 0.409 & 0.543 \\
\hline PTF11bof & 0.487 & 0.429 & 0.545 \\
\hline iPTF14afv & 0.441 & 0.367 & 0.515 \\
\hline iPTF13adw & 0.528 & 0.476 & 0.58 \\
\hline iPTF13acz & 0.586 & 0.532 & 0.641 \\
\hline PTF10qsc & 0.549 & 0.46 & 0.638 \\
\hline PTF10egs & 0.42 & 0.33 & 0.51 \\
\hline iPTF13dkj & 0.392 & 0.318 & 0.467 \\
\hline PTF10cmj & 0.497 & 0.351 & 0.644 \\
\hline PTF12cks & 0.555 & 0.507 & 0.603 \\
\hline iPTF14aaf & 0.518 & 0.449 & 0.587 \\
\hline PTF10fxp & 0.398 & 0.308 & 0.488 \\
\hline PTF12juu & 0.372 & 0.221 & 0.523 \\
\hline PTF10hld & 0.497 & 0.428 & 0.567 \\
\hline PTF12hwb & 0.443 & 0.287 & 0.6 \\
\hline iPTF14aik & 0.283 & 0.073 & 0.494 \\
\hline iPTF16gef & 0.297 & 0.145 & 0.449 \\
\hline PTF10hei & 0.446 & 0.374 & 0.519 \\
\hline PTF10qqt & 0.519 & 0.403 & 0.635 \\
\hline PTF10fym & 0.398 & 0.32 & 0.476 \\
\hline PTF10yux & 0.322 & 0.217 & 0.428 \\
\hline PTF10ifj & 0.54 & 0.444 & 0.637 \\
\hline iPTF14axt & 0.361 & 0.282 & 0.44 \\
\hline iPTF13cwq & 0.465 & 0.395 & 0.535 \\
\hline PTF10abws & 0.429 & 0.304 & 0.554 \\
\hline iPTF14yy & 0.376 & 0.314 & 0.437 \\
\hline iPTF13bmn & 0.39 & 0.276 & 0.503 \\
\hline PTF10oum & 0.382 & 0.312 & 0.452 \\
\hline iPTF13cd & 0.429 & 0.363 & 0.495 \\
\hline
\end{tabular}




\begin{tabular}{|c|c|c|c|}
\hline iPTF16gmw & 0.344 & 0.22 & 0.468 \\
\hline iPTF13dbp & 0.248 & 0.225 & 0.271 \\
\hline PTF12ibh & 0.439 & 0.326 & 0.551 \\
\hline PTF10ivt & 0.388 & 0.271 & 0.505 \\
\hline iPTF13dnh & 0.552 & 0.498 & 0.606 \\
\hline iPTF13cxn & 0.469 & 0.399 & 0.539 \\
\hline PTF11rke & 0.481 & 0.387 & 0.575 \\
\hline PTF12sz & 0.454 & 0.388 & 0.52 \\
\hline iPTF13anh & 0.441 & 0.373 & 0.51 \\
\hline iPTF14aqs & 0.321 & 0.045 & 0.598 \\
\hline iPTF13ag & 0.471 & 0.416 & 0.526 \\
\hline PTF10cko & 0.494 & 0.4 & 0.588 \\
\hline PTF10fxl & 0.556 & 0.513 & 0.599 \\
\hline iPTF13bjb & 0.431 & 0.348 & 0.513 \\
\hline PTF10kiw & 0.526 & 0.407 & 0.645 \\
\hline iPTF13bdb & 0.35 & 0.124 & 0.577 \\
\hline iPTF13dfa & 0.294 & 0.228 & 0.36 \\
\hline PTF10kee & 0.396 & 0.311 & 0.481 \\
\hline PTF10kdg & 0.421 & 0.325 & 0.517 \\
\hline PTF10abkt & 0.32 & 0.17 & 0.47 \\
\hline iPTF14dcd & 0.474 & 0.433 & 0.515 \\
\hline PTF10qkv & 0.469 & 0.419 & 0.519 \\
\hline iPTF13cyy & 0.487 & 0.419 & 0.555 \\
\hline PTF10qly & 0.527 & 0.437 & 0.617 \\
\hline PTF11kaw & 0.491 & 0.399 & 0.583 \\
\hline iPTF13adv & 0.463 & 0.411 & 0.515 \\
\hline iPTF16gua & 0.281 & 0.188 & 0.374 \\
\hline PTF10ujl & 0.414 & 0.259 & 0.569 \\
\hline PTF12gaz & 0.308 & 0.06 & 0.557 \\
\hline PTF11ao & 0.413 & 0.227 & 0.6 \\
\hline PTF10rbp & 0.49 & 0.395 & 0.584 \\
\hline PTF11hfu & 0.588 & 0.451 & 0.725 \\
\hline PTF10feg & 0.561 & 0.483 & 0.64 \\
\hline PTF10goo & 0.498 & 0.44 & 0.555 \\
\hline iPTF13cor & 0.346 & 0.27 & 0.423 \\
\hline iPTF13bun & 0.443 & 0.247 & 0.639 \\
\hline iPTF14anq & 0.408 & 0.203 & 0.614 \\
\hline PTF11rrq & 0.403 & 0.326 & 0.48 \\
\hline iPTF13caz & 0.423 & 0.355 & 0.492 \\
\hline PTF10twd & 0.587 & 0.504 & 0.67 \\
\hline iPTF13cow & 0.477 & 0.39 & 0.563 \\
\hline iPTF13daw & 0.339 & 0.268 & 0.411 \\
\hline PTF10one & 0.37 & 0.302 & 0.438 \\
\hline iPTF16sw & 0.49 & 0.385 & 0.595 \\
\hline iPTF13dni & 0.468 & 0.407 & 0.529 \\
\hline PTF11ilj & 0.537 & 0.406 & 0.667 \\
\hline PTF10qyx & 0.375 & 0.307 & 0.444 \\
\hline iPTF13adm & 0.449 & 0.384 & 0.514 \\
\hline PTF11qve & 0.516 & 0.419 & 0.614 \\
\hline iPTF16ig & 0.405 & 0.288 & 0.522 \\
\hline PTF10aaju & 0.467 & 0.395 & 0.539 \\
\hline iPTF16fht & 0.208 & 0.073 & 0.344 \\
\hline PTF10xup & 0.473 & 0.411 & 0.534 \\
\hline PTF12kta & 0.402 & 0.306 & 0.499 \\
\hline PTF12csi & 0.493 & 0.423 & 0.563 \\
\hline PTF10lxp & 0.429 & 0.384 & 0.475 \\
\hline PTF10wyq & 0.432 & 0.319 & 0.546 \\
\hline iPTF14fyt & 0.805 & 0.624 & 0.987 \\
\hline PTF10gjx & 0.48 & 0.423 & 0.537 \\
\hline PTF12dxm & 0.388 & 0.146 & 0.63 \\
\hline iPTF13dad & 0.475 & 0.426 & 0.524 \\
\hline PTF12lgq & 0.497 & 0.288 & 0.707 \\
\hline
\end{tabular}


Papadogiannakis S. et al.

$\begin{array}{lccc}\text { PTF12vr } & 0.474 & 0.388 & 0.56 \\ \text { PTF10czc } & 0.454 & 0.393 & 0.514 \\ \text { iPTF13ceq } & 0.46 & 0.32 & 0.6 \\ \text { PTF10ufj } & 0.47 & 0.39 & 0.551 \\ \text { PTF10goq } & 0.484 & 0.418 & 0.55 \\ \text { PTF10qwm } & 0.539 & 0.447 & 0.631 \\ \text { iPTF13ddg } & 0.462 & 0.414 & 0.51 \\ \text { iPTF13akl } & 0.487 & 0.388 & 0.586 \\ \text { PTF12gmu } & 0.337 & 0.174 & 0.5 \\ \text { PTF10trp } & 0.497 & 0.272 & 0.723 \\ \text { PTF11dwn } & 0.534 & 0.461 & 0.607 \\ \text { PTF12gqh } & 0.443 & 0.357 & 0.529 \\ \text { PTF10abou } & 0.513 & 0.425 & 0.601 \\ \text { PTF10gsp } & 0.044 & 0.028 & 0.06 \\ \text { iPTF14bjp } & 0.514 & 0.46 & 0.567 \\ \text { iPTF13aig } & 0.553 & 0.499 & 0.608 \\ \text { PTF12gaw } & 0.432 & 0.307 & 0.557 \\ \text { iPTF16fhz } & 0.274 & 0.16 & 0.388\end{array}$

This paper has been typeset from a $\mathrm{T}_{\mathrm{E}} \mathrm{X} / \mathrm{LA} \mathrm{T}_{\mathrm{E}} \mathrm{X}$ file prepared by the author. 